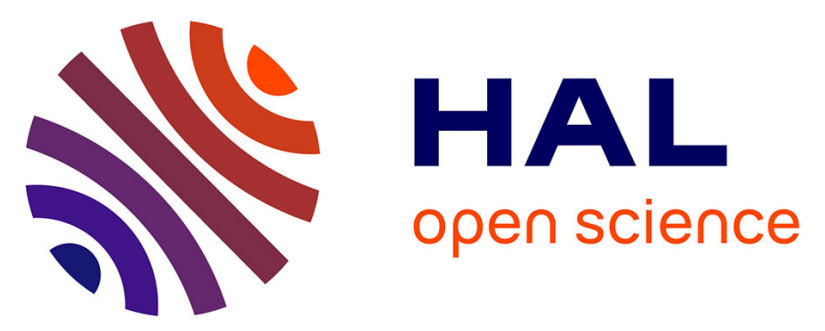

\title{
Asymptotic Analysis of Area Spectral Efficiency and Energy Efficiency in PPP Networks with SLNR Precoder
}

Ahmad Mahbubul Alam, Philippe Mary, Jean-Yves Baudais, Xavier Lagrange

\section{- To cite this version:}

Ahmad Mahbubul Alam, Philippe Mary, Jean-Yves Baudais, Xavier Lagrange. Asymptotic Analysis of Area Spectral Efficiency and Energy Efficiency in PPP Networks with SLNR Precoder. IEEE Transactions on Communications, 2017, 65 (7), pp.3172 - 3185. 10.1109/TCOMM.2017.2699192 . hal-01551410

\author{
HAL Id: hal-01551410 \\ https://hal.science/hal-01551410
}

Submitted on 30 Jun 2017

HAL is a multi-disciplinary open access archive for the deposit and dissemination of scientific research documents, whether they are published or not. The documents may come from teaching and research institutions in France or abroad, or from public or private research centers.
L'archive ouverte pluridisciplinaire HAL, est destinée au dépôt et à la diffusion de documents scientifiques de niveau recherche, publiés ou non, émanant des établissements d'enseignement et de recherche français ou étrangers, des laboratoires publics ou privés. 


\title{
Asymptotic Analysis of Area Spectral Efficiency and Energy Efficiency in PPP Networks with SLNR Precoder
}

\author{
Ahmad Mahbubul Alam, Philippe Mary, Member, IEEE, Jean-Yves Baudais, Member, IEEE, \\ Xavier Lagrange, Member, IEEE
}

\begin{abstract}
This paper aims at characterizing the energy efficiency - area spectral efficiency (EE-ASE) tradeoff in random geometry networks with multiple-antenna arrays at base stations (BSs). In particular, ASE and EE are studied w.r.t. the transmit power when BSs use a signal-to-leakage-plus-noise ratio (SLNR) precoder. When the static power consumption cannot be neglected, EE behaves linearly w.r.t. ASE before a sharp decreasing of EE due to the interference-limited characteristic of the network. Our contribution relies on the derivation of a closedform expression for ASE with SLNR precoder in the asymptotic regime, i.e. when the number of antennas and users grows to infinity, using stochastic geometry. We derive EE from a linear power consumption model afterwards. Unlike conventional SLNR precoders, the average signal-to-interference-plus-noise ratio and the leakage to other cells are considered in a geometry dependent network. Extensive Monte Carlo simulations show that despite the asymptotic nature of the theoretical analysis, the closed-form expressions are tight w.r.t. simulations even for moderate number of antennas and users. Hence, the analysis can be used for realistic network performance analysis.
\end{abstract}

Index Terms-multiple-input multiple-output (MIMO), precoder, signal-to-leakage-plus-noise ratio (SLNR), Poisson point process (PPP), energy efficiency-area spectral efficiency (EEASE)

\section{INTRODUCTION}

\section{A. Context}

Densification of cellular network appears as a suitable answer to meet the increasing data rate demand of the customers. Network densification means increasing the number of base stations (BSs), and number of BS antennas. Although the rollout of densification has undoubtedly a positive effect on the spectral efficiency (SE), the static power consumption is also increased due to the large amount of infrastructure. Therefore, it is important to know how SE and energy efficiency (EE) scale with the system parameters, e.g. number of BS antennas, BS-user density ratio, to determine the appropriate amount of infrastructure when static power consumption is taken into account. Maximizing EE and SE are conflicting objectives and

A. M. Alam and P. Mary are with INSA, IETR, UMR 6164, 35708 Rennes, France. Email: ahmad-mahbubul.alam@insa-rennes.fr, philippe.mary@insarennes.fr

J.-Y. Baudais is with CNRS, IETR, UMR 6164, 35708 Rennes, France. Email: jean-yves.baudais@insa-rennes.fr

$\mathrm{X}$. Lagrange is with IMT Atlantique, IRISA D2, Rennes, FRANCE. Email: xavier.lagrange@imt-atlantique.fr

This work has been presented in part in IEEE SPAWC 2016, Edinburgh, UK.

Manuscript received November XX, 2016; revised XXX, XXX. a fundamental tradeoff exists between these metrics when only the BS transmit power is considered [1]. Fundamental EE-SE tradeoff for several multiplexing technologies, i.e. time division multiple access, frequency division multiple access and superposition coding, has been studied in [2] using Poisson point processes (PPP). Global network energy consumption considering macro and small cells deployment with different densities has been investigated in [3]. The authors found that the optimal energy consumption is function of the ratio between the densities of macro and small cells, and small cell deployment is preferable in conjunction with turn off some macro BSs when the ratio is lower than a threshold. However, the static power consumption is not taken into account in these works. Since the BS static power consumption cannot be neglected, the tradeoff between EE and SE considering a practical power consumption model has been a growing subject of interest in recent years [4]-[8].

Space division multiple access (SDMA) enables the communication between $\mathrm{BS}$ and users in the same time-frequency resources. SDMA can achieve higher rates compared to communication schemes operating in separate time-frequency resources, which are not optimal from an information-theoretic point of view [9]. Serving multiple users in the same timefrequency resources increases interference, and precoding is used in downlink to mitigate this interference. Implementing the capacity achieving precoder based on superposition coding is complex and therefore linear precoding techniques, e.g. zero-forcing (ZF), signal-to-leakage-plus-noise ratio (SLNR), minimum mean square error (MMSE), are preferred. Although the ZF precoder nulls the intra-cell interference [10]-[12], it is not designed to limit the inter-cell interference. Moreover, the $\mathrm{ZF}$ precoder imposes a restriction on the minimum number of transmit antennas at BS [13]. On the other hand, MMSE performs better than $\mathrm{ZF}$ in both high and low signal-to-noise ratio regime. However, MMSE precoder is generally difficult to handle theoretically [14].

Authors in [15], [16] have proposed SLNR as an alternative optimization metric for designing the precoder in multiuser multiple-input multiple output (MIMO) downlink communications. Equivalence between MMSE and SLNR precoders under symmetric scenarios where channels to the users are equally strong has been shown in [14], [17]. Although the network geometry is random in our work, we consider the SLNR precoder for its closed-to-optimal performance and simplicity. SLNR does not impose any restriction on the number of BS 
antennas in contrast to ZF solution [15] and also considers the influence of noise.

\section{B. Related Works}

Performance of MIMO in a multi-cell environment is quite different from that in a single cell environment due to intercell interference. The works in [15], [16] did not consider multi-cell environment and also ignored the network geometry, i.e. distance dependent path loss, while computing the SLNR precoder. An equivalent optimization metric, i.e. signal-togenerating-interference-plus-noise ratio (SGINR), is used by authors in [18]. The maximization of SLNR or SGINR is a generalized eigenvalue problem and hence named as the generalized eigenvalue-based beamformer in [19]. Based on uplink-downlink duality, virtual SINR maximizing beamforming is considered in [20], which is equivalent to the SLNR precoder. Although the works in [18]-[20] considered multicell environment, the results were obtained by performing Monte Carlo simulations. In contrast to the previous works, the novelty of this paper is to develop a tight approximation of the expressions of ASE and EE using SLNR precoder in a multi-cell network when BSs and users are modelled with homogeneous Poisson point processes. The expressions provide useful insights on how ASE and EE vary in multiantenna random networks and can be calculated much faster than performing Monte Carlo simulations.

Stochastic geometry has been used for the performance analysis of cellular networks intensively during the past decade with the work of Baccelli, Andrews or Haenggi among others [21], [22]. The authors of [10] have used the stochastic geometry tool to study the relation between ASE and different system parameters considering a ZF precoder, which is found to perform worse than the SLNR precoder in terms of the energy efficiency-area spectral efficiency (EE-ASE) tradeoff [23]. Most of the previous works dealing with stochastic geometry to analyze the network performances, have considered either a maximal ratio transmission or ZF precoder [10]-[12], [24]. In our recent work [23], the achievable EE-ASE tradeoff obtained with ZF and SLNR for a PPP network has been studied. SLNR reveals to achieve a better EE-ASE tradeoff than ZF due to the fact that the latter does not deal with the inter-cell interference and also decreases the received desired signal power when the channel vectors of the selected user subset are not orthogonal. However, the results in [23] have been obtained thanks to extensive Monte Carlo simulations. In contrast to [23], a theoretical analysis considering the SLNR precoder for PPP network is proposed in this paper, which has never been done in literature to the best of our knowledge.

The average signal-to-interference-plus-noise ratio (SINR) is calculated applying four theorems, which are stated in Section V. The expression of the average SINR includes Stieltjes transform of non-random limit spectral distribution (LSD) functions, resulted from random empirical spectral distribution (ESD) functions of random Hermitian matrices with large dimensions following the theorem provided by Silverstein in [25], and also the derivative of Stieltjes transform. The result given in [25] has been first used in [26] to achieve a closed form expression of the asymptotic mean of the signal-tointerference ratio at the output of an MMSE receiver in the uplink of a single cell. The works in [27], [28] provided an approximation of the SINR distribution for an MMSE receiver in a MIMO system using the same asymptotic technique. The authors in [29] applied the Silverstein's Theorem at the output of an MMSE receiver in a single-hop ad-hoc wireless network, and calculated the mean and variance of the SINR. Although all these prior works follow similar mathematical approaches based on the Silverstein's Theorem in [25] to obtain Stieltjes transform, they have considered different network models compared to this work. Moreover, these works have considered the uplink that resulted in a simpler SINR expressions compared to ours since we assume downlink in our work.

\section{Contributions}

The main contributions of this paper are summarized as follows.

- A tight approximation of the expression of the mean SINR for a user in the downlink of a multi-user (MU) multiple-input single-output (MISO) cellular network with random topology is derived when the max-SLNR precoder is used. The result is achieved by combining some fundamental results from random matrix theory (RMT) to PPP. Based on this, ASE and EE are derived afterwards. These are observed to be very closed to the ones obtained from Monte Carlo simulations. The analytical findings developed in this paper provide useful insights in addition to saving the time to run extensive Monte Carlo simulations. Theoretical analysis of ASE and EE considering the SLNR precoder for a PPP network is the major contribution of this work.

- EE is shown to increase first linearly w.r.t. ASE, i.e. when the transmit power increases, until an optimal point before decreasing sharply due to the interference-limited regime. Moreover, we show that although ASE always increases with BS density and number of BS antennas, this is not always the case for EE which depends on the increase in ASE w.r.t. the increase on the static circuit power consumption. Besides, SLNR precoder is shown to have a significant performance gain compared to $\mathrm{ZF}$ precoder, typically used in a PPP cellular network, in terms of achievable EE and ASE.

The remaining of the paper is organised as follows. Section II presents the system model, Section III revisits the SLNR and ZF precoder in the random network geometry environment. Section IV describes the network performance metrics, i.e. ASE and EE, Section V presents the calculation of mean SINR and Section VI provides the numerical and simulation results. Finally, conclusions and further works are drawn in Section VII.

Notations: $(\cdot)^{H}$ denotes the Hermitian (transposeconjugate) operation while $(\cdot)^{T}$ simply the transposition, $\operatorname{diag}\left(x_{1}, \cdots, x_{n}\right)$ is a square diagonal matrix filled by the elements $\left\{x_{i}\right\}_{i=1, \cdots, n}$. 


\section{SYSTEM MODEL}

A downlink MU-MISO cellular network is considered where BSs are equipped with $M$ transmit antennas and users have a single receive antenna. BSs and users are modeled by two independent PPPs with density $\lambda_{b}$ and $\lambda_{u}$ respectively and users are connected to the nearest BS. Some BSs do not transmit any signal, and are called inactive, since they do not have any user to serve due to the independent locations of BSs and users. All BSs have the same transmit power, which is equally divided among the active users in the cell. The whole system bandwidth is allocated to each user, and hence users experience both intra and inter-cell interferences. A widely used linear power consumption model is considered for BSs [30], [31]:

$$
P_{\mathrm{BS}}=\frac{1}{\eta} P_{t}+M P_{c}+P_{0},
$$

where $\eta \in(0,1]$ is the power amplifier efficiency, $P_{t}$ is the transmit power, $P_{c}$ is the circuit power corresponding to one RF chain, $P_{0}$ represents the non-transmission power for baseband processing, battery backup, cooling, etc. and $P_{\mathrm{BS}}$ is the total BS power consumption.

Rayleigh flat fading channels between BS and users are considered and perfect channel state information is assumed to be available at each BS. The signal received by the $k$-th user from the typical 0-th BS is given by

$$
y_{k}=\sqrt{\frac{P_{t} r_{0 k}^{-\alpha}}{u_{0}}} \mathbf{h}_{0 k}^{H} \mathbf{w}_{0 k} x_{0 k}+\sum_{(i, j) \in \mathcal{A}} \sqrt{\frac{P_{t} r_{i k}^{-\alpha}}{u_{i}}} \mathbf{h}_{i k}^{H} \mathbf{w}_{i j} x_{i j}+n_{k},
$$

where $\mathcal{A}=\left\{\{i, j\} \in \mathbb{N}^{2} \mid i=0 \wedge j \neq k \vee i \neq 0, \forall j\right\}, r_{i k}$ is the distance from the $i$-th BS to the $k$-th user, $\{i, k\} \in \mathbb{N}^{2}, \alpha$ is the path loss exponent, $P_{t}$ the transmit power, $u_{i}$ is the number of users in the $i$-th cell and $n_{k}$ the additive white Gaussian noise (AWGN) with zero mean and variance $\sigma^{2}$ for user $k$. Moreover, $\mathbf{h}_{i k} \sim \mathcal{C N}\left(0, \mathbf{I}_{M}\right)$ is an $M \times 1$ vector representing the complex Gaussian distributed channel between the $i$-th BS and the $k$-th user where $\mathbf{I}_{M}$ is an $M \times M$ identity matrix. In addition, $\mathbf{w}_{i j} \in \mathbb{C}^{M \times 1}, x_{i j}$ are the precoding vector and the transmitted symbol respectively for the $j$-th user in the $i$-th cell with $\mathrm{E}\left[\left|x_{i j}\right|^{2}\right]=1$.

Following the approach in [10], BSs are divided into subsets denoted as $\Phi_{u}$, with $u \in\left[0, u_{\max }\right]$, and each BS in the subset $\Phi_{u}$ serves $u$ users. The maximum number of users served simultaneously in a cell is set to $u_{\max }$ in order to control the interference. The number of users in different cells is assumed to be independent, and each BS group $\Phi_{u}$ follows an homogeneous PPP distribution with density $\lambda_{b} p_{N}(u)$, where $p_{N}(u)$ is the probability mass function (PMF) of the number of active users in a cell. The calculation of $p_{N}(u)$ requires the exact size distribution of the Poisson-Voronoi typical cell, which has been given in [32]. However, since the expression is challenging to compute numerically, a curve-fitted equation has been proposed in [33]. Using this equation, PMF of the number of active users in a cell can be expressed as in [10]:

$$
p_{N}(u)=\left\{\begin{array}{l}
\frac{\mu^{\mu} \Gamma(u+\mu) \rho^{-u}}{\Gamma(\mu) u !\left(\frac{1}{\rho}+\mu\right)^{u+\mu}} \quad 0 \leq u \leq u_{\max }-1 \\
\sum_{n=u_{\max }^{\infty}}^{\infty} \frac{\mu^{\mu} \Gamma(n+\mu) \rho^{-n}}{\Gamma(\mu) n !\left(\frac{1}{\rho}+\mu\right)^{n+\mu}} \quad u=u_{\max }
\end{array}\right.
$$

where $n$ is the number of users available in a cell, $\mu=3.5$ is a constant obtained through data fitting [33], $\rho=\frac{\lambda_{b}}{\lambda_{u}}$ is the BS-user density ratio, and $\Gamma(\cdot)$ is the gamma function. When $n \leq u_{\max }, n$ users are served by BS, while BS randomly chooses $u_{\max }$ users to serve when $n>u_{\max }$. Due to the limitation of active users to $u_{\max }$, their locations become correlated, which is very challenging to handle. In order to make the problem tractable, the simplifying assumption that the set of active users is the sum of independent PPPs with density $\lambda_{b} u p_{N}(u)$ with $u \in\left[1, u_{\max }\right]$ is made. Since the sum of independent PPPs is another PPP, the set of active users is considered as a PPP with density $\lambda_{a u}=\sum_{u=1}^{u_{\max }} \lambda_{b} u P_{N}(u)$. The network is considered to be a circular disc of radius $R$, and total number of active users in this network is $K$ which is a random variable in a given area. However we have $\lim _{R \rightarrow \infty} K(R)=\lambda_{a u} \pi R^{2}$ when the network size grows to infinity. By grouping the interfering BSs into subsets, the SINR of the $k$-th user can be written as

$$
\gamma\left(u_{0}\right)=\frac{\frac{P_{t} r_{0 k}^{-\alpha}}{u_{0}}\left|\mathbf{h}_{0 k}^{H} \mathbf{w}_{0 k}\right|^{2}}{\sum_{j \neq k} \frac{P_{t} r_{0 k}^{-\alpha}}{u_{0}}\left|\mathbf{h}_{0 k}^{H} \mathbf{w}_{0 j}\right|^{2}+\sum_{u=1}^{u_{\max }} \sum_{i \in \Phi_{u} \backslash\{0\}} \sum_{j=1}^{u} \frac{P_{t} r_{i k}^{-\alpha}}{u}\left|\mathbf{h}_{i k}^{H} \mathbf{w}_{i j}\right|^{2}+\sigma^{2}} .
$$

At denominator, the first and second terms refer to the intra and inter-cell interferences respectively.

\section{SLNR AND ZF PRECODING SCHEMES}

\section{A. SLNR Precoder}

The SLNR is defined as the ratio of the received signal power at the desired user to the interference created by the desired signal on the other terminals, also known as leakage, plus the noise power of the desired user. For user $k$, SLNR can be expressed as

$$
\begin{aligned}
\text { SLNR } & =\frac{\frac{P_{t} r_{0 k}^{-\alpha}}{u_{0}} \mathbf{w}_{0 k}^{H} \mathbf{h}_{0 k} \mathbf{h}_{0 k}^{H} \mathbf{w}_{0 k}}{\sum_{j \neq k} \frac{P_{t} r_{0 j}^{-\alpha}}{u_{0}} \mathbf{w}_{0 k}^{H} \mathbf{h}_{0 j} \mathbf{h}_{0 j}^{H} \mathbf{w}_{0 k}+\sigma^{2}} \\
& =\frac{r_{0 k}^{-\alpha} \mathbf{w}_{0 k}^{H} \mathbf{h}_{0 k} \mathbf{h}_{0 k}^{H} \mathbf{w}_{0 k}}{\mathbf{w}_{0 k}^{H} \overline{\mathbf{H}}_{0 k} \overline{\mathbf{D}}_{0 k} \overline{\mathbf{H}}_{0 k}^{H} \mathbf{w}_{0 k}+\frac{\sigma^{2} u_{0}}{P_{t}}},
\end{aligned}
$$

where $\overline{\mathbf{H}}_{0 k}=\left[\mathbf{h}_{01}, \cdots, \mathbf{h}_{0(k-1)}, \mathbf{h}_{0(k+1)}, \cdots, \mathbf{h}_{0 K}\right]$ and $\overline{\mathbf{D}}_{0 k}=\operatorname{diag}\left(r_{01}^{-\alpha}, \cdots, r_{0(k-1)}^{-\alpha}, r_{0(k+1)}^{-\alpha}, \cdots, r_{0 K}^{-\alpha}\right)$ represent the concatenated fading channels and a square diagonal matrix filled by the path losses from the 0-th BS to the active users in the network, except the $k$-th user. With average power constraint, the SLNR maximization for user $k$ is:

$$
\mathbf{w}_{0 k}=\arg \max _{\mathbf{w}_{0 k} \in \mathbb{C}^{M \times 1}} \frac{\mathbf{w}_{0 k}^{H} \mathbf{h}_{0 k} \mathbf{h}_{0 k}^{H} \mathbf{w}_{0 k}}{\mathbf{w}_{0 k}^{H}\left(\overline{\mathbf{H}}_{0 k} \overline{\mathbf{D}}_{0 k} \overline{\mathbf{H}}_{0 k}^{H}+\frac{\sigma^{2} u_{0}}{P_{t}\left\|\mathbf{w}_{0 k}\right\|^{2}} \mathbf{I}_{M}\right) \mathbf{w}_{0 k}}
$$


where $\|\cdot\|$ represents the $L_{2}$ vector norm. When $M \rightarrow \infty$, the optimization problem in (6) can be proved to be equivalent to

$$
\mathbf{w}_{0 k}=\arg \max _{\mathbf{w}_{0 k} \in \mathbb{C}^{M \times 1}} \frac{\mathbf{w}_{0 k}^{H} \mathbf{h}_{0 k} \mathbf{h}_{0 k}^{H} \mathbf{w}_{0 k}}{\mathbf{w}_{0 k}^{H}\left(\overline{\mathbf{H}}_{0 k} \overline{\mathbf{D}}_{0 k} \overline{\mathbf{H}}_{0 k}^{H}+\frac{\sigma^{2} u_{0}}{P_{t}} \mathbf{I}_{M}\right) \mathbf{w}_{0 k}}
$$

subject to $\mathrm{E}\left[\left\|\mathbf{w}_{0 k}\right\|^{2}\right]=1$.

Indeed, defining $\mathbf{w}_{0 k}=\left[w_{0 k 1}, w_{0 k 2}, \cdots, w_{0 k M}\right]^{T}$, we can write

$$
\mathrm{E}\left[\left\|\mathbf{w}_{0 k}\right\|^{2}\right]=\mathrm{E}\left[\sum_{i}^{M} w_{0 k i}^{2}\right]=\sum_{i}^{M} \mathrm{E}\left[w_{0 k i}^{2}\right]=M \mathrm{E}\left[w_{0 k i}^{2}\right]=1
$$

where the final step in (8) is obtained considering that $w_{0 k i}$ are iid with the same second order moment. Let the random variable $x_{i}=M w_{0 k i}^{2}$ with the mean value independent of $M$ and equal to 1 , i.e. the mean value of $w_{0 k i}^{2}$ decreases with $M$. Then, according to the law of large numbers (LLN),

$$
\left\|\mathbf{w}_{0 k}\right\|^{2}=\frac{1}{M} \sum_{i}^{M} x_{i} \stackrel{\text { LLN }}{\longrightarrow} \mathrm{E}\left[x_{i}\right]=1 .
$$

For (9) be true, the variance of $x_{i}$ should be finite, which is assumed to be true and seems a reasonable assumption. From (8) and (9), we can write $\lim _{M \rightarrow \infty}\left\|\mathbf{w}_{0 k}\right\|^{2}=\mathrm{E}\left[\left\|\mathbf{w}_{0 k}\right\|^{2}\right]$ and hence replace the problem in $(6)$ by the problem in (7).

Using the generalized Rayleigh quotient theorem, the solution of (7) is the eigenvector corresponding to the maximum eigenvalue [34]:

$\mathbf{w}_{0 k} \propto \max$ eigenvect $\left(\overline{\mathbf{H}}_{0 k} \overline{\mathbf{D}}_{0 k} \overline{\mathbf{H}}_{0 k}^{H}+\frac{\sigma^{2} u_{0}}{P_{t}} \mathbf{I}_{M}\right)^{-1} \mathbf{h}_{0 k} \mathbf{h}_{0 k}^{H}$.

The resulting SLNR is equal to the maximum eigenvalue $\lambda_{\max }$ [17]. Any vector $\mathbf{g}_{0 k}$, which is in the eigenspace corresponding to $\lambda_{\max }$, satisfies the following eigenvector equation:

$$
\left(\overline{\mathbf{H}}_{0 k} \overline{\mathbf{D}}_{0 k} \overline{\mathbf{H}}_{0 k}^{H}+\frac{\sigma^{2} u_{0}}{P_{t}} \mathbf{I}_{M}\right)^{-1} \mathbf{h}_{0 k} \mathbf{h}_{0 k}^{H} \mathbf{g}_{0 k}=\lambda_{\max } \mathbf{g}_{0 k} .
$$

Inserting a nonzero complex scalar $\delta_{0 k}=\mathbf{h}_{0 k}^{H} \mathbf{g}_{0 k}$ into (11), $\mathrm{g}_{0 k}$ can be written as

$$
\mathbf{g}_{0 k}=\frac{\delta_{0 k}}{\lambda_{\max }}\left(\overline{\mathbf{H}}_{0 k} \overline{\mathbf{D}}_{0 k} \overline{\mathbf{H}}_{0 k}^{H}+\frac{\sigma^{2} u_{0}}{P_{t}} \mathbf{I}_{M}\right)^{-1} \mathbf{h}_{0 k} .
$$

Imposing the power constraint, solution to (7) is

$$
\begin{aligned}
& \left(\overline{\mathbf{H}}_{0 k} \overline{\mathbf{D}}_{0 k} \overline{\mathbf{H}}_{0 k}^{H}+\frac{\sigma^{2} u_{0}}{P_{t}} \mathbf{I}_{M}\right)^{-1} \mathbf{h}_{0 k}
\end{aligned}
$$

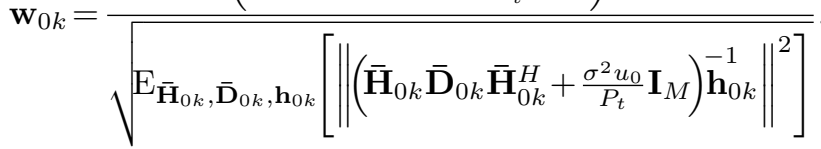

Monte Carlo simulations have been performed on the CDF of SLNR when the precoding vector $\mathbf{w}_{0 k}$ is as in (13), and when it is obtained by solving (7) and imposing an instantaneous power constraint, i.e. $\left\|\mathbf{w}_{0 k}\right\|^{2}=1$ which is equivalent to remove the expectation in the denominator of (13). Simulations have been conducted considering several PPP network realizations and channel fading, and the CDF of SLNR is evaluated for a typical user located at center of the

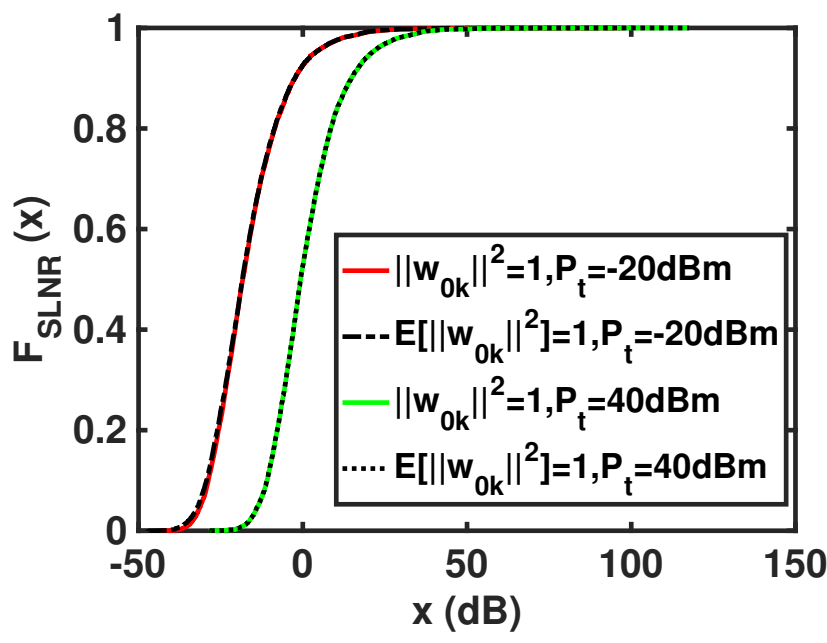

Figure 1: CDF of the SLNR at $M=10$

network. Parameters for the plots are set to $M=10, \alpha=4$, $\sigma^{2}=-97.5 \mathrm{dBm}, \lambda_{u}=5 \cdot 10^{-4} \mathrm{~m}^{-2}, \rho=0.0077$. A perfect match of the CDFs with the instantaneous and the average power constraint is observed in Fig. 1. This suggests that $\mathrm{E}\left[\left\|\mathbf{w}_{0 k}\right\|^{2}\right]$ can be used as a good approximation of $\left\|\mathbf{w}_{0 k}\right\|^{2}$ even for $M$ as small as 10 .

\section{B. ZF Precoder}

ZF precoder is defined such that $\mathbf{H}_{0}^{H} \mathbf{G}_{0}=\mathbf{I}_{u_{0}}$ [35], where $\mathbf{H}_{0}=\left[\mathbf{h}_{01}, \cdots, \mathbf{h}_{0 k}, \cdots, \mathbf{h}_{0 u_{0}}\right] \in \mathbb{C}^{M \times u_{0}}$ and $\mathbf{G}_{0} \in \mathbb{C}^{M \times u_{0}}$ respectively represent the concatenated channels and nonnormalized precoding vectors for the users in cell 0 . Nonnormalized precoding matrix $\mathbf{G}_{0}$ can be chosen as the pseudoinverse of $\mathbf{H}_{0}$, which for $u_{0} \leq M$ can be written as [23]

$$
\mathbf{G}_{0}=\mathbf{H}_{0}\left[\mathbf{H}_{0}^{H} \mathbf{H}_{0}\right]^{-1} .
$$

Normalized precoding vector for the $k$-th user is given by

$$
\mathbf{w}_{0 k}=\frac{\left[\mathbf{G}_{0}\right]_{k}}{\left\|\left[\mathbf{G}_{0}\right]_{k}\right\|},
$$

where $\left[\mathbf{G}_{0}\right]_{k}$ is the $k$-th column of $\mathbf{G}_{0}$.

\section{Performance Metrics}

\section{A. Area Spectral Efficiency}

ASE is defined as the average sum of the ergodic rate of users per unit area (u.a.). Ergodic throughput of a cell with $u_{0}$ active users is given by

$$
R_{\mathrm{BS}}\left(u_{0}\right)=u_{0} R_{u}\left(u_{0}\right),
$$

where $R_{u}\left(u_{0}\right)$ is the ergodic rate of a typical user when there are $u_{0}$ active users in the cell. The ergodic throughput of a typical BS averaged over the number of active users can be written as

$$
R_{\mathrm{BS}}=\mathrm{E}_{u_{0}}\left[u_{0} R_{u}\left(u_{0}\right)\right]=\sum_{u_{0}=1}^{u_{\max }} u_{0} R_{u}\left(u_{0}\right) p_{N}\left(u_{0}\right) .
$$


Using (17), ASE in b/s/Hz/u.a. can be written as

$$
\eta_{\mathrm{ASE}}=\lambda_{b} \sum_{u_{0}=1}^{u_{\max }} u_{0} R_{u}\left(u_{0}\right) p_{N}\left(u_{0}\right) .
$$

Three different random processes, i.e. two independent PPPs governing users and BSs locations, and fading channels are taken into account in the system model. To calculate the ergodic rate of the $k$-th user, we first average the rate over the SINR conditioned on distance to the connected BS and then average over this random quantity. Therefore, the ergodic SE of the $k$-th user with $u_{0}$ users in the cell is

$$
R_{u}\left(u_{0}\right)=\mathrm{E}_{r_{0 k}}\left[\mathrm{E}_{\gamma}\left[\log _{2}\left(1+\gamma\left(u_{0}\right)\right) \mid r_{0 k}\right]\right],
$$

where $\mathrm{E}_{\gamma}$ and $\mathrm{E}_{r_{0 k}}$ denote the expectations over the SINR, and the distance of the $k$-th user from the connected BS respectively. Note that (19) holds only when $x_{i j}$ are Gaussian and when transmitters have the knowledge of CSI of active users in the network, which is assumed in order to implement the SLNR precoder. The authors in [36] assumed the aggregated interference-plus-noise to be Gaussian distributed, but without the strain of receiver's knowledge about the fading of each individual interference term. This enabled the authors to express the ergodic SE as a function of the local-average SINR and involving the expectation over the intended fading channel only. However, this is a lower bound of the ergodic SE that could be achieved if the receiver had the knowledge about each individual interference term. The authors are then able to perform the spatial average of the function of the local SINR if this latter is known. In this work, SLNR precoder is used and then the channel state information of active users in the network are required at transmitters in order to realize the benefits of SLNR precoding in MU-MISO system. Therefore, the approach followed in [36] is not applicable to our case.

Now, (19) can be calculated using coverage probability [21] or moment generating function approaches [37]. Both these methods necessitate finding the distribution of the terms $\left|\mathbf{h}_{0 k}^{H} \mathbf{w}_{0 k}\right|^{2},\left|\mathbf{h}_{0 k}^{H} \mathbf{w}_{0 j}\right|^{2}$ and $\left|\mathbf{h}_{i k}^{H} \mathbf{w}_{i j}\right|^{2}$, which are difficult to obtain. Using the Jensen's inequality, we first search for an upper bound of $R_{u}\left(u_{0}\right)$,

$$
R_{u}\left(u_{0}\right) \leq \mathrm{E}_{r_{0 k}}\left[\log _{2}\left(1+\mathrm{E}_{\gamma}\left[\gamma\left(u_{0}\right) \mid r_{0 k}\right]\right)\right]=\hat{R}_{u}\left(u_{0}\right) .
$$

Secondly, $\mathrm{E}_{\gamma}\left[\gamma\left(u_{0}\right) \mid r_{0 k}\right]$ is assumed to be close to the ratio of the average quantities in (4) as it has been done in [38] and is given in (21) at the top of the next page.

While the numerator in (21) represents the average desired power, the first and second terms at denominator refer to the average intra and inter-cell interference powers respectively. Note that all these average powers are calculated conditioned on $r_{0 k}$. An upper bound of $R_{u}\left(u_{0}\right)$ can be written as

$$
\hat{R}_{u}\left(u_{0}\right)=\int_{r_{0 k} \geq 0}^{\infty} \log _{2}\left(1+\mathrm{E}_{\gamma}\left[\gamma\left(u_{0}\right) \mid r_{0 k}\right]\right) f_{r_{0 k}}\left(r_{0 k}\right) d r_{0 k},
$$

where $f_{r_{0 k}}\left(r_{0 k}\right)$ is the probability density function (PDF) of the distance of the $k$-th user from its connected BS, and is given by [21]

$$
f_{r_{0 k}}\left(r_{0 k}\right)=e^{-\lambda_{b} \pi r_{0 k}^{2}} 2 \pi \lambda_{b} r_{0 k}
$$

With (18), an upper bound of ASE can be written as

$$
\hat{\eta}_{\mathrm{ASE}}=\lambda_{b} \sum_{u_{0}=1}^{u_{\max }} u_{0} \hat{R}_{u}\left(u_{0}\right) p_{N}\left(u_{0}\right) .
$$

\section{B. Energy Efficiency}

$\mathrm{EE}$ is another important performance metric for cellular networks, which is defined as the ratio of ASE to the average power consumption per u.a. To determine the average power consumption per u.a., only non-transmission power consumption is considered for inactive BSs. Therefore, the average power consumption per u.a. can be written as

$$
P_{A}=\lambda_{b}\left(1-p_{N}(0)\right)\left(\frac{1}{\eta} P_{t}+M P_{c}\right)+\lambda_{b} P_{0}
$$

and hence an upper bound of EE is

$$
\hat{\eta}_{\mathrm{EE}}=\frac{\hat{\eta}_{\mathrm{ASE}}}{P_{A}},
$$

which is obtained using (24) and (25). The computation of ASE and EE requires to calculate an approximation of $\mathrm{E}_{\gamma}\left[\gamma\left(u_{0}\right) \mid r_{0 k}\right]$ as suggested in (21). The simulation results in Section VI will validate the approximation done on the average SINR.

\section{Calculation of $\mathrm{E}_{\gamma}\left[\gamma\left(u_{0}\right) \mid r_{0 k}\right]$}

\section{A. Average Desired Power Conditioned on $r_{0 k}$}

Using (13), we can write

$$
\begin{aligned}
& \left|\mathbf{h}_{0 k}^{H} \mathbf{w}_{0 k}\right|^{2}= \\
& \frac{\left|\mathbf{h}_{0 k}^{H}\left(\overline{\mathbf{H}}_{0 k} \overline{\mathbf{D}}_{0 k} \overline{\mathbf{H}}_{0 k}^{H}+\frac{\sigma^{2} u_{0}}{P_{t}} \mathbf{I}_{M}\right)^{-1} \mathbf{h}_{0 k}\right|^{2}}{\mathrm{E}_{\overline{\mathbf{H}}_{0 k}, \overline{\mathbf{D}}_{0 k}, \mathbf{h}_{0 k}\left[\left\|\left(\overline{\mathbf{H}}_{0 k} \overline{\mathbf{D}}_{0 k} \overline{\mathbf{H}}_{0 k}^{H}+\frac{\sigma^{2} u_{0}}{P_{t}} \mathbf{I}_{M}\right) \mathbf{h}_{0 k}\right\|^{2}\right]}} .
\end{aligned}
$$

We manipulate (27) in order to apply Theorem 1.1 in [25] and obtain

$$
\begin{aligned}
& \left|\mathbf{h}_{0 k}^{H} \mathbf{w}_{0 k}\right|^{2}= \\
& \left|\mathbf{h}_{0 k}^{H}\left(\frac{1}{M} \overline{\mathbf{H}}_{0 k} M^{\frac{\alpha}{2}} \overline{\mathbf{D}}_{0 k} \overline{\mathbf{H}}_{0 k}^{H}+\frac{M^{\frac{\alpha}{2}-1} \sigma^{2} u_{0}}{P_{t}} \mathbf{I}_{M}\right)^{-1} \mathbf{h}_{0 k}\right|^{2} \\
& \mathrm{E}_{\overline{\mathbf{H}}_{0 k}, \overline{\mathbf{D}}_{0 k}, \mathbf{h}_{0 k}\left[\left.\left\|\left(\frac{1}{M} \overline{\mathbf{H}}_{0 k} M^{\frac{\alpha}{2}} \overline{\mathbf{D}}_{0 k} \overline{\mathbf{H}}_{0 k}^{H}+\frac{M^{\frac{\alpha}{2}-1} \sigma^{2} u_{0}}{P_{t}} \mathbf{I}_{M}\right) \mathbf{h}_{0 k}\right\|^{2}\right|^{2}\right]} .
\end{aligned}
$$

Considering the eigen decomposition: $\frac{1}{M} \overline{\mathbf{H}}_{0 k} M^{\frac{\alpha}{2}} \overline{\mathbf{D}}_{0 k} \overline{\mathbf{H}}_{0 k}^{H}=$ $\overline{\mathbf{U}}_{0 k} \overline{\boldsymbol{\Lambda}}_{0 k} \overline{\mathbf{U}}_{0 k}^{H}$ where $\overline{\mathbf{U}}_{0 k} \in \mathbb{C}^{M \times M}$ is a unitary matrix and $\bar{\Lambda}_{0 k} \in \mathbb{R}_{+}^{M \times M}$ is a diagonal matrix containing the eigenvalues, and writing $\mathbf{t}_{0 k}=\overline{\mathbf{U}}_{0 k}^{H} \mathbf{h}_{0 k}$, (28) can be expressed as

$$
\left|\mathbf{h}_{0 k}^{H} \mathbf{w}_{0 k}\right|^{2}=\frac{\left|\mathbf{t}_{0 k}^{H}\left(\overline{\boldsymbol{\Lambda}}_{0 k}+\frac{M^{\frac{\alpha}{2}-1} \sigma^{2} u_{0}}{P_{t}} \mathbf{I}_{M}\right)^{-1} \mathbf{t}_{0 k}\right|^{2}}{\mathrm{E}_{\mathbf{t}_{0 k}, \overline{\boldsymbol{\Lambda}}_{0 k}}\left[\mathbf{t}_{0 k}^{H}\left(\overline{\boldsymbol{\Lambda}}_{0 k}+\frac{M^{\frac{\alpha}{2}-1} \sigma^{2} u_{0}}{P_{t}} \mathbf{I}_{M}\right)^{-2} \mathbf{t}_{0 k}\right]} .
$$




$$
\mathrm{E}_{\gamma}\left[\gamma\left(u_{0}\right) \mid r_{0 k}\right] \simeq \frac{\mathrm{E}_{\mathbf{h}_{0 k}, \mathbf{w}_{0 k}}\left[\frac{P_{t} r_{0 k}^{-\alpha}}{u_{0}}\left|\mathbf{h}_{0 k}^{H} \mathbf{w}_{0 k}\right|^{2} \mid r_{0 k}\right]}{\mathrm{E}_{\mathbf{h}_{0 k}, \mathbf{w}_{0 j}}\left[\left.\sum_{j \neq k} \frac{P_{t} r_{0 k}^{-\alpha}}{u_{0}}\left|\mathbf{h}_{0 k}^{H} \mathbf{w}_{0 j}\right|^{2}\right|_{r_{0 k}}\right]+\mathrm{E}_{\mathbf{h}_{i k}, \mathbf{w}_{i j}, r_{i k}}\left[\left.\sum_{u=1}^{u_{\max }} \sum_{i \in \Phi_{u} \backslash\{0\}} \frac{P_{t} r_{i k}^{-\alpha}}{u} \sum_{j=1}^{u}\left|\mathbf{h}_{i k}^{H} \mathbf{w}_{i j}\right|^{2}\right|_{r_{0 k}}\right]+\sigma^{2}}
$$

With $\mathbf{t}_{0 k}=\left[t_{0 k 1}, t_{0 k 2}, t_{0 k 3}, \cdots, t_{0 k M}\right]^{T}$ and $\overline{\mathbf{\Lambda}}_{0 k}=$ $\operatorname{diag}\left(\bar{\lambda}_{0 k 1}, \bar{\lambda}_{0 k 2}, \bar{\lambda}_{0 k 3}, \cdots, \bar{\lambda}_{0 k M}\right)$, (29) can be written as

$$
\left|\mathbf{h}_{0 k}^{H} \mathbf{w}_{0 k}\right|^{2}=\frac{\left(\sum_{l=1}^{M} \frac{\left|t_{0 k l}\right|^{2}}{\bar{\lambda}_{0 k l}+\frac{M^{\frac{\alpha}{2}-1} \sigma^{2} u_{0}}{P_{t}}}\right)^{2}}{\mathrm{E}_{\mathbf{t}_{0 k}, \overline{\mathbf{\Lambda}}_{0 k}}\left[\sum_{l=1}^{M} \frac{\left|t_{0 k l}\right|^{2}}{\left(\bar{\lambda}_{0 k l}+\frac{M^{\frac{\alpha}{2}-1} \sigma^{2} u_{0}}{P_{t}}\right)^{2}}\right]} .
$$

Since $\mathbf{w}_{0 k}$ does not include $r_{0 k}$, the expectation of $\left|\mathbf{h}_{0 k}^{H} \mathbf{w}_{0 k}\right|^{2}$ conditioned on $r_{0 k}$ is the same as its unconditional expectation. Therefore, using (30), we can write

$$
\begin{aligned}
\mathrm{E}_{\mathbf{h}_{0 k}, \mathbf{w}_{0 k}}\left[\left.\left|\mathbf{h}_{0 k}^{H} \mathbf{w}_{0 k}\right|^{2}\right|_{r_{0 k}}\right] & = \\
& \frac{\mathrm{E}_{\mathbf{t}_{0 k}, \overline{\mathbf{\Lambda}}_{0 k}}\left[\left(\sum_{l=1}^{M} \frac{\left|t_{0 k l}\right|^{2}}{\bar{\lambda}_{0 k l}+\frac{M^{\frac{\alpha}{2}-1} \sigma^{2} u_{0}}{P_{t}}}\right)^{2}\right]}{\mathrm{E}_{\mathbf{t}_{0 k}, \overline{\mathbf{\Lambda}}_{0 k}}\left[\sum_{l=1}^{M} \frac{\left|t_{0 k l}\right|^{2}}{\left(\bar{\lambda}_{0 k l}+\frac{M^{\frac{\alpha}{2}-} \sigma^{2} u_{0}}{P_{t}}\right)^{2}}\right]} .
\end{aligned}
$$

The constraint $\mathrm{E}\left[\left\|\mathbf{w}_{0 k}\right\|^{2}\right]=1$ allows us to write (31) since the expectation of denominator is already present in (28). Numerator of (31) can be written as

$$
\begin{aligned}
& \mathrm{E}_{\mathbf{t}_{0 k}, \overline{\mathbf{\Lambda}}_{0 k}} {\left[\left(\sum_{l=1}^{M} \frac{\left|t_{0 k l}\right|^{2}}{\bar{\lambda}_{0 k l}+\frac{M^{\frac{\alpha}{2}-1} \sigma^{2} u_{0}}{P_{t}}}\right)^{2}\right]=} \\
&\left(\mathrm{E}_{\mathbf{t}_{0 k}, \overline{\mathbf{\Lambda}}_{0 k}}\left[\sum_{l=1}^{M} \frac{\left|t_{0 k l}\right|^{2}}{\bar{\lambda}_{0 k l}+\frac{M^{\frac{\alpha}{2}-1} \sigma^{2} u_{0}}{P_{t}}}\right]\right)^{2}+ \\
& \operatorname{var}_{\mathbf{t}_{0 k}, \overline{\mathbf{\Lambda}}_{0 k}}\left[\sum_{l=1}^{M} \frac{\left|t_{0 k l}\right|^{2}}{\bar{\lambda}_{0 k l}+\frac{M^{\frac{\alpha}{2}-1} \sigma^{2} u_{0}}{P_{t}}}\right] .
\end{aligned}
$$

We introduce two theorems to calculate (31). While Theorem 1 is used to calculate the first term in the right hand side (RHS) of (32), Theorem 2 is used to calculate the second term in the RHS of (32) and the denominator in (31). Let us start with a definition:

Definition 1. (Asymptotic regime) Let $R$ be the radius of the circular area centered at BS of interest and $\gamma_{a u} \in \mathbb{R}^{+} a$ constant. The asymptotic regime (a.r.) refers to the condition $\lim _{K, M \rightarrow+\infty} \frac{K}{M}=\gamma_{a u}$ with $\lim _{R \rightarrow+\infty} \frac{K(R)}{\lambda_{a u} \pi R^{2}}=1$ and will be referred as $\stackrel{a . r}{\longrightarrow}$ in the rest of the paper.

Theorem 1. Considering the 0-th BS,

$$
\mathrm{E}_{\mathbf{t}_{0 k}, \overline{\mathbf{\Lambda}}_{0 k}}\left[\frac{1}{M} \sum_{l=1}^{M} \frac{\left|t_{0 k l}\right|^{2}}{\bar{\lambda}_{0 k l}-z}\right] \stackrel{a \cdot r}{\longrightarrow} \bar{m}_{0 k}(z)
$$

with $\bar{m}_{0 k}(z)$ is the unique, non-negative real solution of the following equation:

$$
\begin{aligned}
& \frac{\pi \csc \left(\frac{2 \pi}{\alpha}\right)}{\bar{m}_{0 k}^{-\frac{2}{\alpha}}(z) \alpha}-\frac{z \bar{m}_{0 k}(z)}{2 \pi \lambda_{a u}}-\frac{\bar{m}_{0 k}(z)}{\alpha-2}\left(\left(\frac{\pi \lambda_{a u}}{\gamma_{a u}}\right)^{-\frac{\alpha}{2}}+\bar{m}_{0 k}(z)\right)^{\frac{2}{\alpha}-1} \times \\
& { }_{2} F_{1}\left(1-\frac{2}{\alpha}, 1-\frac{2}{\alpha}, 2-\frac{2}{\alpha}, \frac{\left(\frac{\pi \lambda_{a u}}{\gamma_{a u}}\right)^{\frac{\alpha}{2}} \bar{m}_{0 k}(z)}{1+\left(\frac{\pi \lambda_{a u}}{\gamma_{a u}}\right)^{\frac{\alpha}{2}} \bar{m}_{0 k}(z)}\right)=\frac{1}{2 \pi \lambda_{a u}}, \text { (34) }
\end{aligned}
$$

where $z=-\frac{M^{\frac{\alpha}{2}-1} \sigma^{2} u_{0}}{P_{t}}, \csc \left(\frac{2 \pi}{\alpha}\right)$ is the cosecant function of $\frac{2 \pi}{\alpha}$, and ${ }_{2} F_{1}(a, b, c, z)$ is the Gauss hypergeometric function [39].

Proof. See Appendix VIII-A.

Theorem 2. Considering the 0-th BS,

$$
\begin{aligned}
\operatorname{var}_{\mathbf{t}_{0 k}, \overline{\mathbf{\Lambda}}_{0 k}}\left[\frac{1}{\sqrt{M}} \sum_{l=1}^{M} \frac{\left|t_{0 k l}\right|^{2}}{\bar{\lambda}_{0 k l}-z}\right] \\
\stackrel{a . r .}{\longrightarrow} \mathrm{E}_{\mathbf{t}_{0 k}, \overline{\mathbf{\Lambda}}_{0 k}}\left[\frac{1}{M} \sum_{l=1}^{M} \frac{\left|t_{0 k l}\right|^{2}}{\left(\bar{\lambda}_{0 k l}-z\right)^{2}}\right] \stackrel{a \cdot r}{\longrightarrow} m_{0 k}^{\prime}(z),
\end{aligned}
$$

where $m_{0 k}^{\prime}(z)$ is the differentiation of $\bar{m}_{0 k}(z)$ w.r.t. $z$ and expressed as

$$
m_{0 k}^{\prime}(z)=\bar{m}_{0 k}(z)\left(\frac{4 \pi^{2} \lambda_{a u} \csc \left(\frac{2 \pi}{\alpha}\right)}{\bar{m}_{0 k}^{1-\frac{2}{\alpha}}(z) \alpha^{2}}-z+Q\right)^{-1}
$$

with $z=-\frac{M^{\frac{\alpha}{2}-1} \sigma^{2} u_{0}}{P_{t}}$ and

$$
\begin{aligned}
& Q=\frac{2 \pi \lambda_{a u} \bar{m}_{0 k}(z)\left(\frac{\pi \lambda_{a u}}{\gamma_{a u}}\right)^{\alpha-1}\left(1+\bar{m}_{0 k}(z)\left(\frac{\pi \lambda_{a u}}{\gamma_{a u}}\right)^{\frac{\alpha}{2}}\right)^{\frac{2}{\alpha}-2} \times}{2 \alpha-2} \times-\frac{2 \pi \lambda_{a u}\left(\frac{\pi \lambda_{a u}}{\gamma_{a u}}\right)^{\frac{\alpha}{2}-1}}{\alpha-2} \times \\
& \left.\quad\left(1+\bar{m}_{0 k}(z)\left(\frac{\pi \lambda_{a u}}{\gamma_{a u}}\right)^{\frac{\alpha}{2}}\right)^{\frac{2}{\alpha}-1} F_{1}\left(1-\frac{2}{\alpha}, 1-\frac{2}{\alpha}, 3-\frac{2}{\alpha}, \frac{\left(\frac{\pi \lambda_{a u}}{\gamma_{a u}}\right)^{\frac{\alpha}{2}} \bar{m}_{0 k}(z)}{1+\left(\frac{\pi \lambda_{a u}}{\gamma_{a u}}\right)^{\frac{\alpha}{2}} \bar{m}_{0 k}(z)}\right)-\frac{2}{\alpha}, \frac{\left(\frac{\pi \lambda_{a u}}{\gamma_{a u}}\right)^{\frac{\alpha}{2}} \bar{m}_{0 k}(z)}{1+\left(\frac{\pi \lambda_{a u}}{\gamma_{a u}}\right)^{\frac{\alpha}{2}} \bar{m}_{0 k}(z)}\right) .
\end{aligned}
$$

Proof. See Appendix VIII-B.

Using (31), (32), and Theorems 1 and 2, the average desired signal power conditioned on $r_{0 k}$ can be expressed as

$$
\begin{aligned}
\mathrm{E}_{\mathbf{h}_{0 k}, \mathbf{w}_{0 k}}\left[\left.\frac{P_{t} r_{0 k}^{-\alpha}}{u_{0}}\left|\mathbf{h}_{0 k}^{H} \mathbf{w}_{0 k}\right|^{2}\right|_{r_{0 k}}\right] & \\
& \stackrel{a . r_{.}}{\longrightarrow} \frac{P_{t} r_{0 k}^{-\alpha}}{u_{0}}\left(1+\frac{M \bar{m}_{0 k}^{2}(z)}{m_{0 k}^{\prime}(z)}\right) .
\end{aligned}
$$




\section{B. Average Inter-cell Interference Power Conditioned on $r_{0 k}$}

We introduce Theorem 3 to calculate the average inter-cell interference power conditioned on $r_{0 k}$.

Theorem 3. Considering the $i$-th interfering BS,

$$
\mathrm{E}_{\mathbf{h}_{i k}, \mathbf{w}_{i j}}\left[\left.\left|\mathbf{h}_{i k}^{H} \mathbf{w}_{i j}\right|^{2}\right|_{r_{i k}, r_{0 k}}\right] \stackrel{a . r}{\longrightarrow} \frac{1}{\left(1+M^{\frac{\alpha}{2}} r_{i k}^{-\alpha} \bar{m}_{i j k}(z)\right)^{2}},
$$

where $\bar{m}_{i j k}(z)$ is the unique, non-negative real solution of the following equation:

$$
\begin{aligned}
& \frac{\pi c s c\left(\frac{2 \pi}{\alpha}\right)}{\bar{m}_{i j k}^{-\frac{2}{\alpha}}(z) \alpha}-\frac{z \bar{m}_{i j k}(z)}{2 \pi \lambda_{a u}}-\frac{\bar{m}_{i j k}(z)}{\alpha-2}\left(\left(\frac{\pi \lambda_{a u}}{\gamma_{a u}}\right)^{-\frac{\alpha}{2}}+\bar{m}_{i j k}(z)\right)^{\frac{2}{\alpha}-1} \times \\
& { }_{2} F_{1}\left(1-\frac{2}{\alpha}, 1-\frac{2}{\alpha}, 2-\frac{2}{\alpha}, \frac{\left(\frac{\pi \lambda_{a u}}{\gamma_{a u}}\right)^{\frac{\alpha}{2}} \bar{m}_{i j k}(z)}{1+\left(\frac{\pi \lambda_{a u}}{\gamma_{a u}}\right)^{\frac{\alpha}{2}} \bar{m}_{i j k}(z)}\right)=\frac{1}{2 \pi \lambda_{a u}} \text { (38) }
\end{aligned}
$$

with $z=-\frac{M^{\frac{\alpha}{2}-1} \sigma^{2} u_{i}}{P_{t}}$.

Proof. See Appendix VIII-C.

From Theorem 3 and by averaging over $r_{i k}$, we state

Theorem 4. The average inter-cell interference power conditioned on $r_{0 k}$ is

$$
\begin{array}{r}
\mathrm{E}_{\mathbf{h}_{i k}, \mathbf{w}_{i j}, r_{i k}}\left[\left.\sum_{u=1}^{u_{\max }} \sum_{i \in \Phi_{u} \backslash\{0\}} \frac{P_{t} r_{i k}^{-\alpha}}{u} \sum_{j=1}^{u}\left|\mathbf{h}_{i k}^{H} \mathbf{w}_{i j}\right|^{2}\right|_{r_{0 k}}\right] \\
\stackrel{a . r_{.}}{\longrightarrow} \sum_{u=1}^{u_{\max }}\left(f_{1}(u)-f_{2}\left(u, r_{0 k}\right)\right) .
\end{array}
$$

where

$f_{1}(u)=\frac{2 \pi \lambda_{b} p_{N}(u) P_{t} M^{1-\frac{\alpha}{2}} \bar{m}_{i j k}^{\frac{\alpha+2}{\alpha}}(z)_{2} F_{1}\left(\frac{2}{\alpha}, \frac{\alpha+2}{\alpha} ; 2+\frac{2}{\alpha} ; 1\right)}{(\alpha+2) \bar{m}_{i j k}^{2}(z)}$

and

$$
f_{2}\left(u, r_{0 k}\right)=\frac{2 \pi \lambda_{b} p_{N}(u) P_{t 2} F_{1}\left(\frac{2}{\alpha}, \frac{\alpha+2}{\alpha} ; 2+\frac{2}{\alpha} ; \frac{1}{\bar{m}_{i j k}(z) M^{\frac{\alpha}{2}} r_{0 k}^{-\alpha}+1}\right)}{M^{\frac{\alpha}{2}-1}\left(\frac{1}{\bar{m}_{i j k}(z)}+M^{\frac{\alpha}{2}} r_{0 k}^{-\alpha}\right)^{\frac{\alpha+2}{\alpha}}(\alpha+2) \bar{m}_{i j k}^{2}(z)}
$$

Proof. See Appendix VIII-D.

\section{Average Intra-cell Interference Power Conditioned on $r_{0 k}$}

Since $\mathbf{h}_{0 k}^{H} \mathbf{w}_{0 j}$ has the same distribution as $\mathbf{h}_{i k}^{H} \mathbf{w}_{i j}$, $\mathrm{E}_{\mathbf{h}_{0 k}, \mathbf{w}_{0 j}}\left[\left.\left|\mathbf{h}_{0 k}^{H} \mathbf{w}_{0 j}\right|^{2}\right|_{r_{0 k}}\right]$ can be computed with (37) substituting $r_{i k}$ and $\bar{m}_{i j k}(z)$ by $r_{0 k}$ and $\bar{m}_{0 j k}(z)$ respectively. According to Lemma 5 in [40], $\bar{m}_{0 j k}(z)$ is equal to $\bar{m}_{0 k}(z)$ for large $M$, which is the unique, non-negative real solution of (34). Since $\mathrm{E}_{\mathbf{h}_{0 k}, \mathbf{w}_{0 j}}\left[\left.\left|\mathbf{h}_{0 k}^{H} \mathbf{w}_{0 j}\right|^{2}\right|_{r_{0 k}}\right]=$ $\mathrm{E}_{\mathbf{h}_{0 k}, \mathbf{w}_{0 j^{\prime}}}\left[\left.\left|\mathbf{h}_{0 k}^{H} \mathbf{w}_{0 j^{\prime}}\right|^{2}\right|_{r_{0 k}}\right] \forall j \neq j^{\prime}$ except $k$, inserting $\bar{m}_{0 j k}(z)=\bar{m}_{0 k}(z)$ and using Theorem 3, the average intracell interference power conditioned on $r_{0 k}$ can be written as

$$
\begin{aligned}
& \mathrm{E}_{\mathbf{h}_{0 k}, \mathbf{w}_{0 j}}\left[\left.\sum_{j \neq k} \frac{P_{t} r_{0 k}^{-\alpha}}{u_{0}}\left|\mathbf{h}_{0 k}^{H} \mathbf{w}_{0 j}\right|^{2}\right|_{r_{0 k}}\right] \\
& \stackrel{a . r .}{\longrightarrow} \frac{P_{t} r_{0 k}^{-\alpha} \frac{\left(u_{0}-1\right)}{u_{0}}}{\left(1+M^{\frac{\alpha}{2}} r_{0 k}^{-\alpha} \bar{m}_{0 k}(z)\right)^{2}} .
\end{aligned}
$$

Using (36), Theorem 4 and (40), (21) can be written as

$$
\begin{aligned}
& \mathrm{E}_{\gamma}\left[\gamma\left(u_{0}\right) \mid r_{0 k}\right] \\
& \stackrel{a . r .}{\rightarrow} \frac{\frac{P_{t} r_{0 k}^{-\alpha}}{u_{0}}\left(1+\frac{M \bar{m}_{0 k}^{2}(z)}{m_{0 k}^{\prime}(z)}\right)}{\frac{P_{t} r_{0 k}^{-\alpha} \frac{\left(u_{0}-1\right)}{u_{0}}}{\left(1+M^{\frac{\alpha}{2}} r_{0 k}^{-\alpha} \bar{m}_{0 k}(z)\right)^{2}}+\sum_{u=1}^{u_{\max }}\left(f_{1}(u)-f_{2}\left(u, r_{0 k}\right)\right)+\sigma^{2}}
\end{aligned}
$$

Despite that Theorems presented above are derived in asymptotic regime, i.e. large $K$ and $M$, they provide accurate predictions on ASE and EE even for moderate values of $M$ as it will be discussed in the next section.

\section{Numerical and Simulation Results}

Monte Carlo simulations are performed to verify the tightness of the expressions of ASE and EE, i.e. (24) and (26) respectively, when SLNR precoder is used for a system with finite dimensions. A circular area, whose radius is such that the average number of users in the network is $N_{u}$, is considered for simulations. Throughout this section, $N_{u}=5000$ except for the case $\rho=0.1$, where $N_{u}=2500$ was used to reduce the simulation time. The users are positioned uniformly in the area, and the typical user is considered to be located at center of the network. Moreover, $u_{\max }=M, \alpha=4$ and $\sigma^{2}=-97.5 \mathrm{dBm}$ will be used throughout this section unless otherwise mentioned. Finally, $P_{c}, P_{0}$ and $\eta$ are set to $35 \mathrm{~W}$, $34 \mathrm{~W}$ and 0.32 respectively [31].

Figs. $2 \mathrm{a}$ and $2 \mathrm{~b}$ draw ASE vs. $P_{t}$ labelled on BS-user density ratios and number of BS antennas respectively. It is observed that ASE is increasing and converging towards a limit when $P_{t}$ exceeds $20 \mathrm{dBm}$. This is because the network is homogeneous (same transmit power) and interference limited, hence ASE saturates when noise power becomes negligible w.r.t. to the interference power. The results also demonstrate that increasing the BS-user density ratio keeping user density constant, i.e. $\lambda_{u}=5 \cdot 10^{-4} \mathrm{~m}^{-2}$, which is equivalent to increase the BS density, or increasing the number of BS antennas always increase ASE.

EE vs. $P_{t}$ is drawn on Figs. $3 \mathrm{a}$ and $3 \mathrm{~b}$ for different BS-user density ratios and number of BS antennas respectively. It is observed that EE is first increasing when $P_{t}$ is increased. However since ASE converges towards a limit for $P_{t}=20 \mathrm{dBm}$, EE decreases for further increase of $P_{t}$. Fig. $3 \mathrm{~b}$ suggests that increasing the number of antennas slightly improves EE over a wide range of transmit power. Although higher number of antennas induces more RF circuit power, i.e. $P_{c}$, this is not dominant over the increase of ASE over the range of $P_{t}$ taken 


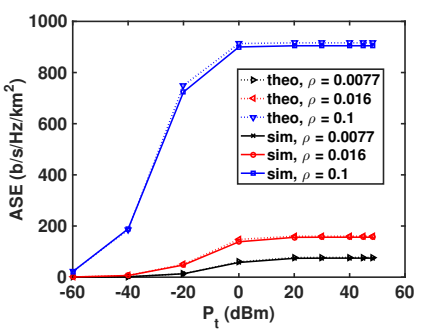

(a) $M=10, \lambda_{u}=5 \cdot 10^{-4} \mathrm{~m}^{-2}$

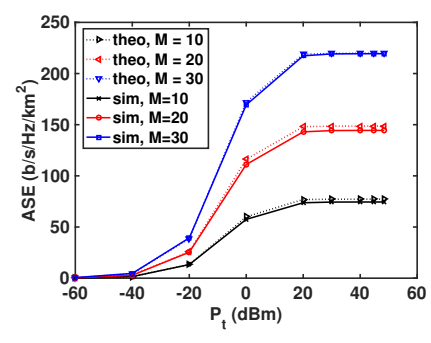

(b) $\rho=0.0077, \lambda_{u}=5 \cdot 10^{-4} \mathrm{~m}^{-2}$

Figure 2: ASE vs. BS transmit power

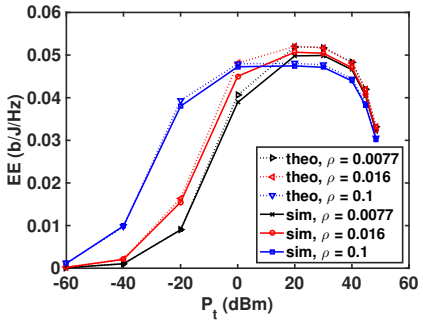

(a) $M=10, \lambda_{u}=5 \cdot 10^{-4} \mathrm{~m}^{-2}$

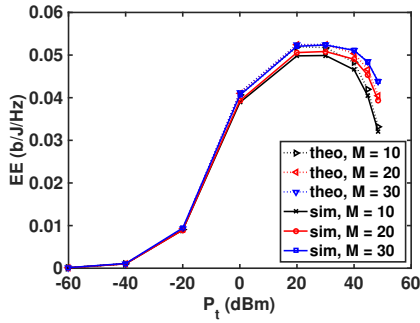

(b) $\rho=0.0077, \lambda_{u}=5 \cdot 10^{-4} \mathrm{~m}^{-2}$

Figure 3: EE vs. BS transmit power

into account. However, this is not always true for arbitrary number of antennas. Deploying more BS antennas increases ASE, but the gain depends on the number of active users per cell and the number of antennas, since they affect the spatial multiplexing gain, the aggregated interference, and the beamforming gain. EE increases only when the increase in ASE dominates the increase of $P_{A}$. On the other hand, it is not energy-efficient to deploy more BSs at $P_{t}$ around $20 \mathrm{dBm}$, Fig. 3a. Indeed, a high roll-out density e.g. $\rho=0.1$, induces a loss in EE compared to a lower BS density, i.e. $\rho=0.016$ and $\rho=0.0077$. ASE increases with BS density, but also the nontransmission power, i.e. $\lambda_{b} P_{0}$, which finally becomes dominant over the increase of ASE for $\rho=0.1$ and then causing the EE decrease.

Figs. $4 \mathrm{a}$ and $4 \mathrm{~b}$ plot the EE-ASE tradeoff for both SLNR and ZF precoder labelled on the BS-user density ratios and number of antennas respectively. We perform Monte Carlo simulations to obtain the EE-ASE tradeoff for the well-known ZF precoder with the same parameters considered for the SLNR precoder, and compare the results with the SLNR precoder. The EE-ASE tradeoff has a large linear part before a sharp decrease when ASE is increased. The linear behavior is due to the significant consumption of the RF circuit and non-transmission powers, i.e. $P_{c}$ and $P_{0}$ respectively. Moreover, since ASE converges towards a limit while EE decreases when $P_{t}$ is increased, a sharp decrease of EE is observed for a slight improvement of ASE, as it has been also observed in regular hexagonal networks [41]. The results also demonstrate that at optimal transmit power, $\rho=0.1$ allows to achieve the best ASE for the SLNR precoder without loosing too much EE compared to other values of $\rho$. In a same way, operating at the optimal transmit power, $M=30$ achieves the best EE-ASE tradeoff compared to $M=10$ or 20 for the SLNR precoder.

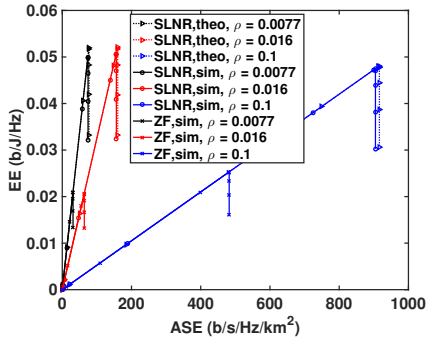

(a) $M=10, \lambda_{u}=5 \cdot 10^{-4} \mathrm{~m}^{-2}$

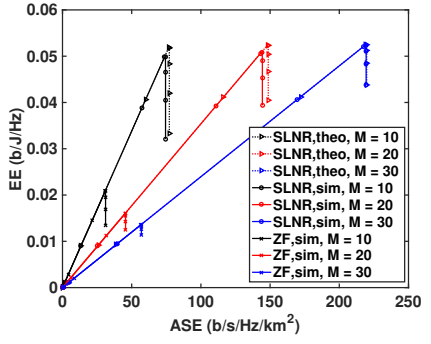

(b) $\rho=0.0077, \lambda_{u}=5 \cdot 10^{-4} \mathrm{~m}^{-2}$
Figure 4: EE vs. ASE

It can also be seen that SLNR precoder has a significant performance gain compared to ZF precoder in terms of achievable EE and ASE. Higher ASE implies a better SINR for the SLNR precoder compared to ZF. This is due to the fact that although ZF precoder nulls the intra-cell interference, it does not account the leakage to other-cell users. Moreover, the total cancellation of the intra-cell interference by $\mathrm{ZF}$ precoding is 2 done at the price of a decrease in the received desired signal power when the channel vectors of the selected user subset are not orthogonal [42]. On the other hand, the multi-cell SLNR precoder achieves a tradeoff between maximizing the received desired signal power of the intended user and minimizing the interference leakage to all other users in order to maximize the SLNR.

Last but not least, our theoretical findings are very tight compared to the simulations results, which suggests that the analytical expressions of ASE and EE, when SLNR precoder is used, can be used as a good approximation of exact values even for $M$ and $K$ as small as 10 and 2500 respectively. Moreover and despite the fact that the analytical expressions for ASE and EE developed from Theorem 1 to 4 are relatively heavy, they are easily computable numerically which is, by far, faster than performing Monte Carlo simulations. Moreover, there is a performance gain over $\mathrm{ZF}$ precoder in terms of EE-ASE tradeoff when SLNR precoder is used.

\section{CONCLUSIONS AND FURTHER WORKS}

This paper has introduced a theoretical framework for approaching the upper bound of ASE in asymptotic regime for PPP networks when SLNR precoder is used by means of random matrix theory. The theoretical expression of the EE-ASE tradeoff has been found to be tight with the results obtained through Monte Carlo simulations, even for moderate values of the number of antennas and users in the network, and for a wide range of system parameters. The results have shown that EE increases linearly as a function of ASE due to the important amount of power wasted in static circuitry. A sharp decrease of EE is observed when transmit power is increased beyond a certain level because of the saturation of ASE at this power. Moreover, although ASE always increases with the BS density and number of antennas, EE depends on the amount of the static power consumption wasted into BS compared to the amount of useful transmit power. 
In further works, the impact of a turn-off strategy on the EE-ASE tradeoff could be studied in conjunction with other precoding techniques, like MMSE precoder.

\section{ACKNOWLEDGEMENT}

This work has received a French government support granted to the CominLabs excellence laboratory and managed by the National Research Agency in the "Investing for the Future" program under reference No. ANR-10-LABX-07-01. The authors would also like to thank the Region Bretagne, France, for its support of this work.

\section{APPENDIX}

\section{A. Proof of Theorem 1}

Let us first derive the CDF of $M^{\frac{\alpha}{2}} r_{0 j}^{-\alpha}$, where $r_{0 j}$ denotes the distance from BS 0 to the $j$-th user. Note that $M^{\frac{\alpha}{2}} r_{0 j}^{-\alpha}$, $\forall j \neq k$ are the diagonal components and also the eigenvalues of $M^{\frac{\alpha}{2}} \overline{\mathbf{D}}_{0 k}$. Considering that BS 0 is positioned at the center of the network, the PDF of $r_{0 j}$ is [28]

$$
f_{r_{0 j}}(x)=\frac{2 x}{R^{2}}
$$

The CDF of $M^{\frac{\alpha}{2}} r_{0 j}^{-\alpha}$ can be written as

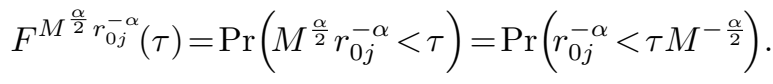

Since $r_{0 j}^{-\alpha}$ is a decreasing function of $r_{0 j}$, using (42), (43) can be written as

$$
\begin{aligned}
F^{M^{\frac{\alpha}{2}} r_{0 j}^{-\alpha}(\tau)} & =1-\operatorname{Pr}\left(r_{0 j}<\left(\tau M^{-\frac{\alpha}{2}}\right)^{\frac{-1}{\alpha}}\right) \\
& =1-\frac{\tau^{\frac{-2}{\alpha}} M}{R^{2}} .
\end{aligned}
$$

Since $\lim _{R \rightarrow \infty} K(R)=\lambda_{a u} \pi R^{2}$, using (44), we can write

$$
\begin{aligned}
\lim _{R \rightarrow \infty} F^{M^{\frac{\alpha}{2}}} r_{0 j}^{-\alpha}(\tau)=\left(1-\frac{\tau^{\frac{-2}{\alpha}} M}{\frac{K}{\pi \lambda_{a u}}}\right) & \stackrel{a . r}{\longrightarrow}\left(1-\frac{\pi \lambda_{a u} \tau^{\frac{-2}{\alpha}}}{\gamma_{a u}}\right) \\
& =F_{l}^{M^{\frac{\alpha}{2}} r_{0 j}^{-\alpha}}(\tau)
\end{aligned}
$$

with $\left(\frac{\pi \lambda_{a u}}{\gamma_{a u}}\right)^{\frac{\alpha}{2}}<\tau<\infty$. Using (45), the derivative of $F_{l}^{M^{\frac{\alpha}{2}} r_{0 j}^{-\alpha}}(\tau)$ can be written as

$$
d F_{l}^{M^{\frac{\alpha}{2}} r_{0 j}^{-\alpha}}(\tau)=\frac{2 \pi \lambda_{a u}}{\alpha \gamma_{a u}} \tau^{-\frac{2}{\alpha}-1} d \tau
$$

The matrix (unitary) of eigenvectors $\overline{\mathbf{U}}_{0 k}$ and the matrix of eigenvalues $\overline{\boldsymbol{\Lambda}}_{0 k}$ are obtained from the eigen decomposition of $\frac{1}{M} \overline{\mathbf{H}}_{0 k} M^{\frac{\alpha}{2}} \overline{\mathbf{D}}_{0 k} \overline{\mathbf{H}}_{0 k}^{H}$ that does not contain $\mathbf{h}_{0 k}$. Since $\mathbf{t}_{0 k}=$ $\mathbb{\mathbf { U }}_{0 k}^{H} \mathbf{h}_{0 k}$, where $\mathbf{h}_{0 k} \sim \mathcal{C N}\left(0, \mathbf{I}_{M}\right)$ then $\mathbf{t}_{0 k} \sim \mathcal{C N}\left(0, \mathbf{I}_{M}\right)$ and hence $\mathbf{t}_{0 k}$ is independent of $\overline{\mathbf{U}}_{0 k}$ and $\overline{\boldsymbol{\Lambda}}_{0 k}$. Since $\left|t_{0 k l}\right|^{2}$ is exponentially distributed with mean 1 , we have

$$
\begin{aligned}
\mathrm{E}_{\mathbf{t}_{0 k}, \bar{\Lambda}_{0 k}} & {\left[\frac{1}{M} \sum_{l=1}^{M} \frac{\left|t_{0 k l}\right|^{2}}{\bar{\lambda}_{0 k l}+\frac{M^{\frac{\alpha}{2}-1} \sigma^{2} u_{0}}{P_{t}}}\right] } \\
& =\frac{1}{M} \sum_{l=1}^{M} \mathrm{E}\left[\left|t_{0 k l}\right|^{2}\right] \mathrm{E}_{\bar{\lambda}_{0 k l}}\left[\frac{1}{\bar{\lambda}_{0 k l}+\frac{M^{\frac{\alpha}{2}-1} \sigma^{2} u_{0}}{P_{t}}}\right] \\
& =\mathrm{E}_{\bar{\lambda}_{0 k l}}\left[\frac{1}{M} \sum_{l=1}^{M} \frac{1}{\bar{\lambda}_{0 k l}+\frac{M^{\frac{\alpha}{2}-1} \sigma^{2} u_{0}}{P_{t}}}\right]
\end{aligned}
$$

The ESD of $\frac{1}{M} \overline{\mathbf{H}}_{0 k} M^{\frac{\alpha}{2}} \overline{\mathbf{D}}_{0 k} \overline{\mathbf{H}}_{0 k}^{H}$ is the proportion of the values of $\bar{\lambda}_{0 k l}$ less than or equal to $x$ which can be expressed as [25]

$$
F^{\overline{\mathbf{\Lambda}}_{0 k}}(x)=\frac{1}{M} \sum_{l=1}^{M} \mathbf{1}_{\bar{\lambda}_{0 k l} \leq x}(x) .
$$

The Stieltjes transform of $F^{\bar{\Lambda}_{0 k}}(x)$ on $\mathbb{C} \backslash \mathbb{R}^{+}$is [29], [39]

$$
m_{F^{\mathbf{\Lambda}_{0 k}}}(z)=\int_{0}^{\infty} \frac{1}{x-z} d F^{\overline{\mathbf{\Lambda}}_{0 k}}(x) .
$$

$F^{\overline{\mathbf{\Lambda}}_{0 k}}$ is random and depends on $\overline{\mathbf{H}}_{0 k} \in \mathbb{C}^{M \times(K-1)}$ and $\overline{\mathbf{D}}_{0 k} \in \mathbb{R}_{+}{ }^{(K-1) \times(K-1)}$. The latter is independent of $\overline{\mathbf{H}}_{0 k}$. Moreover, $\mathrm{E}\left[\overline{\mathbf{H}}_{0 k 11}-\mathrm{E}\left[\overline{\mathbf{H}}_{0 k 11}\right]\right]^{2}=1$ where $\overline{\mathbf{H}}_{0 k 11}$ denotes the element of the first column and first row of the matrix

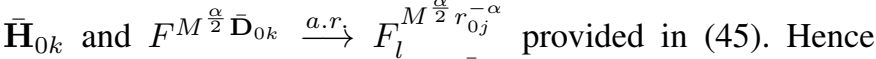
according to Silverstein [25], $F^{\overline{\mathbf{\Lambda}}_{0 k}}$ converges in distribution to a non-random function $F_{l}^{\overline{\boldsymbol{\Lambda}}_{0 k}}$. Moreover, Stieltjes transform of $F_{l}^{\overline{\boldsymbol{\Lambda}}_{0 k}}$ denoted by $m_{F_{l}{ }^{\bar{\Lambda}_{0 k}}}(z)$ satisfies [25]

$$
z m_{F_{l} \overline{\boldsymbol{\Lambda}}_{0 k}}(z)+1=m_{F_{l}} \overline{\mathbf{\Lambda}}_{0 k}(z) \gamma_{a u} \int_{0}^{\infty} \frac{\tau d F_{l}^{M^{\frac{\alpha}{2}} r_{0 j}^{-\alpha}}(\tau)}{1+\tau m_{F_{l} \overline{\mathbf{\Lambda}}_{0 k}}(z)} .
$$

Since the elements of $\bar{\Lambda}_{0 k}$ are positive, the integrand in (49) is bounded and positive for negative values of $z$ hence $F^{\overline{\boldsymbol{\Lambda}}_{0 k}} \rightarrow$ $F_{l}^{\overline{\boldsymbol{\Lambda}}_{0 k}}$ implies $m_{F^{\bar{\Lambda}_{0 k}}}(z) \rightarrow m_{F_{l}} \overline{\boldsymbol{\Lambda}}_{0 k}(z)$ which is also nonrandom [26]. Therefore, the term inside the expectation in the RHS of (47) is $m_{F_{l} \bar{\Lambda}_{0 k}}(z)$ evaluated at $z=-\frac{M^{\frac{\alpha}{2}-1} \sigma^{2} u_{0}}{P_{t}}$ in the asymptotic regime. Therefore, (47) can be written as

$$
\mathrm{E}_{\mathbf{t}_{0 k}, \overline{\boldsymbol{\Lambda}}_{0 k}}\left[\frac{1}{M} \sum_{l=1}^{M} \frac{\left|t_{0 k l}\right|^{2}}{\bar{\lambda}_{0 k l}+\frac{M^{\frac{\alpha}{2}-1} \sigma^{2} u_{0}}{P_{t}}}\right] \stackrel{a . r}{\longrightarrow} m_{F_{l} \overline{\mathbf{\Lambda}}_{0 k}}(z) .
$$

Writing $m_{F_{l} \bar{\Lambda}_{0 k}}=\bar{m}_{0 k}$ for notational simplicity, and using (46), RHS of (50) can be written as

$$
\begin{aligned}
\bar{m}_{0 k}(z) \gamma_{a u} & \int_{0}^{\infty} \frac{\tau d F_{l}^{M^{\frac{\alpha}{2}}} r_{0 j}^{-\alpha}(\tau)}{1+\tau \bar{m}_{0 k}(z)} \\
& =\bar{m}_{0 k}(z) \gamma_{a u} \int_{\left(\frac{\pi \lambda_{a u}}{\gamma_{a u}}\right)^{\frac{\alpha}{2}}}^{\infty} \frac{\frac{2 \pi \lambda_{a u} \tau^{\frac{-2}{\alpha}}}{\alpha \gamma_{a u}}}{1+\tau \bar{m}_{0 k}(z)} d \tau \\
& =\frac{\bar{m}_{0 k}(z)}{\frac{\alpha}{2 \pi \lambda_{a u}}} \int_{\left(\frac{\pi \lambda_{a u}}{\gamma_{a u}}\right)^{\frac{\alpha}{2}}}^{\infty} \frac{\tau^{\frac{-2}{\alpha}}}{1+\tau \bar{m}_{0 k}(z)} d \tau .
\end{aligned}
$$


Applying lemma 1 from [29], (52) can be written as

$$
\begin{aligned}
\bar{m}_{0 k}(z) \gamma_{a u} \int_{0}^{\infty} \frac{\tau d F_{l}^{M \frac{\alpha}{2}} r_{0 j}^{-\alpha}(\tau)}{1+\tau \bar{m}_{0 k}(z)} \\
=\frac{2 \pi^{2} \lambda_{a u} \csc \left(\frac{2 \pi}{\alpha}\right)}{\bar{m}_{0 k}^{-\frac{2}{\alpha}}(z) \alpha}-\frac{2 \pi \lambda_{a u} \bar{m}_{0 k}(z)\left(\frac{\pi \lambda_{a u}}{\gamma_{a u}}\right)^{\frac{\alpha}{2}-1}}{\alpha-2} \times \\
\left(1+\bar{m}_{0 k}(z)\left(\frac{\pi \lambda_{a u}}{\gamma_{a u}}\right)^{\frac{\alpha}{2}}\right)^{\frac{2}{\alpha}-1} \times \\
{ }_{2} F_{1}\left(1-\frac{2}{\alpha}, 1-\frac{2}{\alpha}, 2-\frac{2}{\alpha}, \frac{\left(\frac{\pi \lambda_{a u}}{\gamma_{a u}}\right)^{\frac{\alpha}{2}} \bar{m}_{0 k}(z)}{1+\left(\frac{\pi \lambda_{a u}}{\gamma_{a u}}\right)^{\frac{\alpha}{2}} \bar{m}_{0 k}(z)}\right) .
\end{aligned}
$$

Substituting (53) into (50) and after tedious but straightforward manipulations, (34) is obtained and the proof is complete.

\section{B. Proof of Theorem 2}

Using the law of total variance [27], [29], we can write

$$
\begin{aligned}
\operatorname{var}_{\mathbf{t}_{0 k}, \overline{\boldsymbol{\Lambda}}_{0 k}} & {\left[\frac{1}{\sqrt{M}} \sum_{l=1}^{M} \frac{\left|t_{0 k l}\right|^{2}}{\bar{\lambda}_{0 k l}+\frac{M^{\frac{\alpha}{2}-1} \sigma^{2} u_{0}}{P_{t}}}\right] } \\
= & \mathrm{E}_{\overline{\boldsymbol{\Lambda}}_{0 k}}\left[\operatorname{var}_{\mathbf{t}_{0 k}}\left[\left.\frac{1}{\sqrt{M}} \sum_{l=1}^{M} \frac{\left|t_{0 k l}\right|^{2}}{\bar{\lambda}_{0 k l}+\frac{M^{\frac{\alpha}{2}-1} \sigma^{2} u_{0}}{P_{t}}}\right|_{\overline{\boldsymbol{\Lambda}}_{0 k}}\right]\right] \\
& +\operatorname{var}_{\overline{\boldsymbol{\Lambda}}_{0 k}}\left[\mathrm{E}_{\mathbf{t}_{0 k}}\left[\left.\frac{1}{\sqrt{M}} \sum_{l=1}^{M} \frac{\left|t_{0 k l}\right|^{2}}{\bar{\lambda}_{0 k l}+\frac{M^{\frac{\alpha}{2}-1} \sigma^{2} u_{0}}{P_{t}}}\right|_{\overline{\boldsymbol{\Lambda}}_{0 k}}\right]\right]
\end{aligned}
$$

Since $\left|t_{0 k l}\right|^{2}$ are iid with mean and variance 1 , (54) can be proved to be

$$
\begin{aligned}
\operatorname{var}_{\mathbf{t}_{0 k}, \bar{\Lambda}_{0 k}} & {\left[\frac{1}{\sqrt{M}} \sum_{l=1}^{M} \frac{\left|t_{0 k l}\right|^{2}}{\bar{\lambda}_{0 k l}+\frac{M^{\frac{\alpha}{2}-1} \sigma^{2} u_{0}}{P_{t}}}\right] } \\
= & \mathrm{E}_{\bar{\Lambda}_{0 k}}\left[\frac{1}{M} \sum_{l=1}^{M}\left(\frac{1}{\bar{\lambda}_{0 k l}+\frac{M^{\frac{\alpha}{2}-1} \sigma^{2} u_{0}}{P_{t}}}\right)^{2}\right] \\
& +M \operatorname{var}_{\bar{\Lambda}_{0 k}}\left[\frac{1}{M} \sum_{l=1}^{M} \frac{1}{\bar{\lambda}_{0 k l}+\frac{M^{\frac{\alpha}{2}-1} \sigma^{2} u_{0}}{P_{t}}}\right]
\end{aligned}
$$

The variance term in RHS in (55) can be expressed as in (56) at the top of the next page. Let note $\tilde{\boldsymbol{\Lambda}}_{0 k}=\left(\overline{\boldsymbol{\Lambda}}_{0 k}+\frac{M^{\frac{\alpha}{2}-1} \sigma^{2} u_{0}}{P_{t}}\right)^{-1}, \quad \tilde{\mathbf{D}}_{0 k}=M^{\frac{\alpha}{2}} \overline{\mathbf{D}}_{0 k}=$ $\operatorname{diag}\left(\tilde{d}_{01}, \cdots, \tilde{d}_{0(k-1)}, \tilde{d}_{0(k+1)}, \tilde{d}_{0 K}\right)$ and following the same steps as in [27], [29] and applying Corollary 1.8 in [43], the probability in (56) can be upper bounded by

$$
\begin{aligned}
& \operatorname{Pr}\left[\left|\frac{1}{M} \operatorname{tr}\left(\tilde{\boldsymbol{\Lambda}}_{0 k}\right)-\mathrm{E}\left[\frac{1}{M} \operatorname{tr}\left(\tilde{\boldsymbol{\Lambda}}_{0 k}\right)\right]\right|\right. \\
& \left.>x \mid \tilde{d}_{01}, \cdots, \tilde{d}_{0(k-1)}, \tilde{d}_{0(k+1)}, \tilde{d}_{0 K}\right] \leq 2 e^{-\frac{M^{2} x^{2}}{2 \delta_{1} \delta_{2} \delta_{3}}},
\end{aligned}
$$

where $\delta_{1}>0$ is the Sobolev inequality constant for the distribution of the entries of $\overline{\mathbf{H}}_{0 k}, \delta_{2}=\frac{27}{64\left(\frac{M^{\frac{\alpha}{2}-1} \sigma^{2} u_{0}}{P_{t}}\right)^{3}}$ is the square of the Lipschitz constant for the function $f(x)=$ $\frac{1}{\frac{M^{\frac{\alpha}{2}-1} \sigma^{2} u_{0}}{P}+x^{2}}$ and $\delta_{3}$ is the largest $\tilde{d}_{0 i}$ which is $M^{\frac{\alpha}{2}} \epsilon^{-\alpha}$ considering that $\epsilon$ is the close-in reference distance. Therefore, $\frac{1}{2 \delta_{1} \delta_{2} \delta_{3}} \geq \frac{64\left(\frac{\sigma^{2} u_{0}}{P_{t}}\right)^{3} M^{\alpha-3}}{54 \delta_{1} \epsilon^{-\alpha}}$ and (57) can be written as

$$
\begin{aligned}
& \operatorname{Pr}\left[\left|\frac{1}{M} \operatorname{tr}\left(\tilde{\boldsymbol{\Lambda}}_{0 k}\right)-\mathrm{E}\left[\frac{1}{M} \operatorname{tr}\left(\tilde{\boldsymbol{\Lambda}}_{0 k}\right)\right]\right|\right. \\
& \left.>x \mid \tilde{d}_{01}, \cdots, \tilde{d}_{0(k-1)}, \tilde{d}_{0(k+1)}, \tilde{d}_{0 K}\right] \leq 2 e^{\frac{-64\left(\frac{\sigma^{2} u_{0}}{P_{t}}\right)^{3} M^{\alpha-1} x^{2}}{54 \delta_{1} \epsilon^{-\alpha}}} .
\end{aligned}
$$

Hence, the variance term in RHS of (55) can be upper bounded by

$$
\begin{aligned}
M \operatorname{var}_{\bar{\Lambda}_{0 k}} & {\left[\frac{1}{M} \sum_{l=1}^{M} \frac{1}{\bar{\lambda}_{0 k l}+\frac{M^{\frac{\alpha}{2}-1} \sigma^{2} u_{0}}{P_{t}}}\right] } \\
& \leq M \int_{0}^{\infty} 4 x e^{\frac{-64\left(\frac{\sigma^{2} u_{0}}{P_{t}}\right)^{3} M^{\alpha-1} x^{2}}{54 \delta_{1} \epsilon^{-\alpha}}} d x=\frac{27 \delta_{1} \epsilon^{-\alpha} M^{2-\alpha}}{16\left(\frac{\sigma^{2} u_{0}}{P_{t}}\right)^{3}} .
\end{aligned}
$$

Since the RHS of (59) goes to zero for $\alpha>2$ when $M \rightarrow \infty$, (55) reduces to

$$
\begin{aligned}
\operatorname{var}_{\mathbf{t}_{0 k}, \bar{\Lambda}_{0 k}} & {\left[\frac{1}{\sqrt{M}} \sum_{l=1}^{M} \frac{\left|t_{0 k l}\right|^{2}}{\bar{\lambda}_{0 k l}+\frac{M^{\frac{\alpha}{2}-1} \sigma^{2} u_{0}}{P_{t}}}\right] } \\
& \stackrel{a . r}{\longrightarrow} E_{\bar{\Lambda}_{0 k}}\left[\frac{1}{M} \sum_{l=1}^{M}\left(\frac{1}{\bar{\lambda}_{0 k l}+\frac{M^{\frac{\alpha}{2}-1} \sigma^{2} u_{0}}{P_{t}}}\right)^{2}\right] .
\end{aligned}
$$

Differentiation of $m_{F_{l}} \bar{\Lambda}_{0 k}(z)$ w.r.t. $z$ is

$$
m_{F_{l}{ }^{\bar{\Lambda}_{0 k}}}(z)=\frac{d}{d z} \int_{0}^{\infty} \frac{d F_{l}^{\overline{\mathbf{\Lambda}}_{0 k}}(x)}{x-z}=\int_{0}^{\infty} \frac{d F_{l}^{\overline{\mathbf{\Lambda}}_{0 k}}(x)}{(x-z)^{2}} .
$$

Using (61), we can write

$$
E_{\bar{\Lambda}_{0 k}}\left[\frac{1}{M} \sum_{l=1}^{M}\left(\frac{1}{\bar{\lambda}_{0 k l}+\frac{M^{\frac{\alpha}{2}-1} \sigma^{2} u_{0}}{P_{t}}}\right)^{2}\right] \stackrel{a . r}{\longrightarrow} m_{0 k}^{\prime}(z),
$$

where $z=-\frac{M^{\frac{\alpha}{2}-1} \sigma^{2} u_{0}}{P_{t}}$, and $m_{F_{1} \bar{\Lambda}_{0 k}}^{\prime}$ is denoted by $m_{0 k}^{\prime}$ for notational simplicity, which can be obtained as expressed in (35). On the other hand, using the fact that $\left|t_{0 k l}\right|^{2}$ is independent of $\frac{1}{\left(\bar{\lambda}_{0 k l}+\frac{M^{\frac{\alpha}{2}-1} \sigma^{2} u_{0}}{P_{t}}\right)^{2}}$, and $\mathrm{E}\left[\left|t_{0 k l}\right|^{2}\right]=1$, we have

$$
\begin{aligned}
\mathrm{E}_{\mathbf{t}_{0 k}, \bar{\Lambda}_{0 k}} & {\left[\frac{1}{M} \sum_{l=1}^{M} \frac{\left|t_{0 k l}\right|^{2}}{\left(\bar{\lambda}_{0 k l}+\frac{M^{\frac{\alpha}{2}-1} \sigma^{2} u_{0}}{P_{t}}\right)^{2}}\right] } \\
& =\frac{1}{M} \sum_{l=1}^{M} \mathrm{E}\left[\left|t_{0 k l}\right|^{2}\right] \mathrm{E}_{\bar{\Lambda}_{0 k}}\left[\frac{1}{\left(\bar{\lambda}_{0 k l}+\frac{M^{\frac{\alpha}{2}-1} \sigma^{2} u_{0}}{P_{t}}\right)^{2}}\right] \\
& =\mathrm{E}_{\bar{\Lambda}_{0 k}}\left[\frac{1}{M} \sum_{l=1}^{M} \frac{1}{\left(\bar{\lambda}_{0 k l}+\frac{M^{\frac{\alpha}{2}-1} \sigma^{2} u_{0}}{P_{t}}\right)^{2}}\right]
\end{aligned}
$$

Combining (60), (62) and (63) completes the proof. 


$$
\operatorname{var}_{\bar{\Lambda}_{0 k}}\left[\frac{1}{M} \sum_{l=1}^{M} \frac{1}{\bar{\lambda}_{0 k l}+\frac{M^{\frac{\alpha}{2}-1} \sigma^{2} u_{0}}{P_{t}}}\right]=\int_{0}^{\infty} 2 x \operatorname{Pr}\left[\left|\frac{1}{M} \operatorname{tr}\left(\left(\overline{\boldsymbol{\Lambda}}_{0 k}+\frac{M^{\frac{\alpha}{2}-1} \sigma^{2} u_{0}}{P_{t}}\right)^{-1}\right)-\mathrm{E}\left[\frac{1}{M} \operatorname{tr}\left(\left(\overline{\boldsymbol{\Lambda}}_{0 k}+\frac{M^{\frac{\alpha}{2}-1} \sigma^{2} u_{0}}{P_{t}}\right)^{-1}\right)\right]\right|>x\right] d x
$$

\section{Proof of Theorem 3}

We can write the precoder for the $j$-th user in the $i$-th cell as

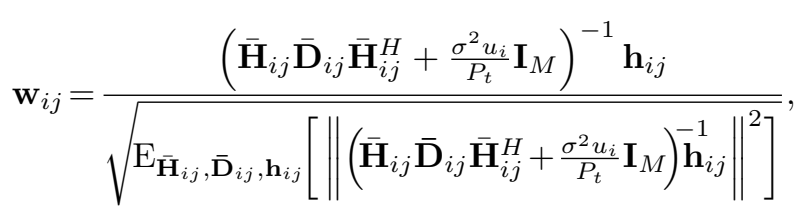

where $\overline{\mathbf{H}}_{i j}=\left[\mathbf{h}_{i 1}, \cdots, \mathbf{h}_{i(j-1)}, \mathbf{h}_{i(j+1)}, \cdots, \mathbf{h}_{i K}\right]$ and $\overline{\mathbf{D}}_{i j}=$ $\operatorname{diag}\left(r_{i 1}^{-\alpha}, \cdots, r_{i(j-1)}^{-\alpha}, r_{i(j+1)}^{-\alpha}, \cdots, r_{i K}^{-\alpha}\right)$ respectively represent the concatenated fading channels and path losses from the $i$-th BS to all active users in the network, except the $j$ th user. Using (64), we can write

$$
\begin{gathered}
\left|\mathbf{h}_{i k}^{H} \mathbf{w}_{i j}\right|^{2}=\frac{\left|\mathbf{h}_{i k}^{H}\left(\overline{\mathbf{H}}_{i j} \overline{\mathbf{D}}_{i j} \overline{\mathbf{H}}_{i j}^{H}+\frac{\sigma^{2} u_{i}}{P_{t}} \mathbf{I}_{M}\right)^{-1} \mathbf{h}_{i j}\right|^{2}}{\mathrm{E}_{\overline{\mathbf{H}}_{i j}, \overline{\mathbf{D}}_{i j}, \mathbf{h}_{i j}}\left[\left\|\left(\overline{\mathbf{H}}_{i j} \overline{\mathbf{D}}_{i j} \overline{\mathbf{H}}_{i j}^{H}+\frac{\sigma^{2} u_{i}}{P_{t}} \mathbf{\mathbf { I }}_{M}\right)^{-1} \mathbf{h}_{i j}\right\|^{2}\right]} \\
=\frac{\left.\left|\mathbf{h}_{i k}^{H}\left(\frac{1}{M} \overline{\mathbf{H}}_{i j k} M^{\frac{\alpha}{2}} \overline{\mathbf{D}}_{i j k} \overline{\mathbf{H}}_{i j k}^{H}+\frac{M^{\frac{\alpha}{2}-1}}{r_{i k}^{\alpha}} \mathbf{h}_{i k} \mathbf{h}_{i k}^{H}-z \mathbf{I}_{M}\right)^{-1}\right|_{i j}^{2}\right|^{2}}{\mathrm{E}_{\overline{\mathbf{H}}_{i j}, \overline{\mathbf{D}}_{i j}, \mathbf{h}_{i j}}\left[\left\|\left(\frac{1}{M} \overline{\mathbf{H}}_{i j} M^{\frac{\alpha}{2}} \overline{\mathbf{D}}_{i j} \overline{\mathbf{H}}_{i j}^{H}-z \mathbf{I}_{M}\right)^{-1} \mathbf{h}_{i j}\right\|^{2}\right]},(65)
\end{gathered}
$$

where $z=-\frac{M^{\frac{\alpha}{2}-1} \sigma^{2} u_{i}}{P_{t}}$, channel matrix $\overline{\mathbf{H}}_{i j k}=$ $\left[\mathbf{h}_{i 1}, \cdots, \mathbf{h}_{i(j-1)}, \mathbf{h}_{i(j+1)}, \cdots, \mathbf{h}_{i(k-1)}, \mathbf{h}_{i(k+1)}, \cdots, \mathbf{h}_{i K}\right]$ and moreover the diagonal matrix $\overline{\mathbf{D}}_{i j k}=$ $\operatorname{diag}\left(r_{i 1}^{-\alpha}, \cdots, r_{i(j-1)}^{-\alpha}, r_{i(j+1)}^{-\alpha}, \cdots, r_{i(k-1)}^{-\alpha}, r_{i(k+1)}^{-\alpha}, \cdots, r_{i K}^{-\alpha}\right)$. Applying lemma 2.5 in [44], (65) can be written as

$$
\begin{aligned}
& \left|\mathbf{h}_{i k}^{H} \mathbf{w}_{i j}\right|^{2} \\
& =\frac{\left|\frac{\mathbf{h}_{i k}^{H}\left(\frac{1}{M} \overline{\mathbf{H}}_{i j k} M^{\frac{\alpha}{2}} \overline{\mathbf{D}}_{i j k} \overline{\mathbf{H}}_{i j k}^{H}-z \mathbf{I}_{M}\right)^{-1} \mathbf{h}_{i j}}{1+\frac{M^{\frac{\alpha}{2}-1}}{r_{i k}^{\alpha}} \mathbf{h}_{i k}^{H}\left(\frac{1}{M} \overline{\mathbf{H}}_{i j k} M^{\frac{\alpha}{2}} \overline{\mathbf{D}}_{i j k} \overline{\mathbf{H}}_{i j k}^{H}-z \mathbf{I}_{M}\right)^{-1} \mathbf{h}_{i k}}\right|^{2}}{\mathrm{E}_{\overline{\mathbf{H}}_{i j}, \overline{\mathbf{D}}_{i j}, \mathbf{h}_{i j}}\left[\left\|\left(\frac{1}{M} \overline{\mathbf{H}}_{i j} M^{\frac{\alpha}{2}} \overline{\mathbf{D}}_{i j} \overline{\mathbf{H}}_{i j}^{H}-z \mathbf{I}_{M}\right)^{-1} \mathbf{h}_{i j}\right\|^{2}\right]} .
\end{aligned}
$$

Since the elements of $\mathbf{h}_{i k}$ are iid complex Gaussian with variance 1 and $\left(\frac{1}{M} \overline{\mathbf{H}}_{i j k} M^{\frac{\alpha}{2}} \overline{\mathbf{D}}_{i j k} \overline{\mathbf{H}}_{i j k}^{H}-z \mathbf{I}_{M}\right)^{-1}$ is independent of $\mathbf{h}_{i k}$, applying lemma 7 in [40] and using (66), we can write

$$
\begin{aligned}
\lim _{M \rightarrow \infty}\left|\mathbf{h}_{i k}^{H} \mathbf{w}_{i j}\right|^{2} & \left|\frac{\mathbf{h}_{i k}^{H}\left(\frac{1}{M} \overline{\mathbf{H}}_{i j k} M^{\frac{\alpha}{2}} \overline{\mathbf{D}}_{i j k} \overline{\mathbf{H}}_{i j k}^{H}-z \mathbf{I}_{M}\right)^{-1} \mathbf{h}_{i j}}{1+\frac{M^{\frac{\alpha}{2}-1}}{r_{i k}^{\alpha}} \operatorname{tr}\left(\left(\frac{1}{M} \overline{\mathbf{H}}_{i j k} M^{\frac{\alpha}{2}} \overline{\mathbf{D}}_{i j k} \overline{\mathbf{H}}_{i j k}^{H}-z \mathbf{I}_{M}\right)^{-1}\right)}\right|^{2} \\
= & \mathrm{E}_{\overline{\mathbf{H}}_{i j}, \overline{\mathbf{D}}_{i j}, \mathbf{h}_{i j}}\left[\left\|\left(\frac{1}{M} \overline{\mathbf{H}}_{i j} M^{\frac{\alpha}{2}} \overline{\mathbf{D}}_{i j} \overline{\mathbf{H}}_{i j}^{H}-z \mathbf{I}_{M}\right) \mathbf{h}_{i j}^{-1}\right\|^{2}\right]
\end{aligned}
$$

Applying the eigen decomposition: $\frac{1}{M} \overline{\mathbf{H}}_{i j k} M^{\frac{\alpha}{2}} \overline{\mathbf{D}}_{i j k} \overline{\mathbf{H}}_{i j k}^{H}=$ $\overline{\mathbf{U}}_{i j k} \overline{\mathbf{\Lambda}}_{i j k} \overline{\mathbf{U}}_{i j k}^{H}$ and $\frac{1}{M} \overline{\mathbf{H}}_{i j} M^{\frac{\alpha}{2}} \overline{\mathbf{D}}_{i j} \overline{\mathbf{H}}_{i j}^{H}=\overline{\mathbf{U}}_{i j} \overline{\mathbf{\Lambda}}_{i j} \overline{\mathbf{U}}_{i j}^{H}$, and inserting $\overline{\mathbf{U}}_{i j k}^{H} \mathbf{h}_{i k}=\mathbf{t}_{i k}, \overline{\mathbf{U}}_{i j k}^{H} \mathbf{h}_{i j}=\mathbf{t}_{N i j}, \overline{\mathbf{U}}_{i j}^{H} \mathbf{h}_{i j}=\mathbf{t}_{D i j}$, (67) can be written as

$$
\begin{aligned}
& \lim _{M \rightarrow \infty}\left|\mathbf{h}_{i k}^{H} \mathbf{w}_{i j}\right|^{2}=\left|\frac{M^{-\frac{\alpha}{2}} \mathbf{t}_{i k}^{H}\left(\overline{\mathbf{\Lambda}}_{i j k}-z \mathbf{I}_{M}\right)^{-1} \mathbf{t}_{N i j}}{M^{-\frac{\alpha}{2}}+\frac{r_{i k}^{-\alpha}}{M} \operatorname{tr}\left(\left(\overline{\boldsymbol{\Lambda}}_{i j k}-z \mathbf{I}_{M}\right)^{-1}\right)}\right|^{2} \\
& \mathrm{E}_{\mathbf{t}_{D i j}, \overline{\mathbf{\Lambda}}_{i j}}\left[\mathbf{t}_{D i j}^{H}\left(\overline{\boldsymbol{\Lambda}}_{i j}-z \mathbf{I}_{M}\right)^{-2} \mathbf{t}_{D i j}\right] \frac{\left|M^{\frac{-\alpha}{2}} \mathbf{t}_{i k}^{H}\left(\overline{\mathbf{\Lambda}}_{i j k}-z \mathbf{I}_{M}\right)^{-1} \mathbf{t}_{N i j}\right|^{2}}{\left(M^{-\frac{\alpha}{2}}+\frac{r_{i k}^{-\alpha}}{M} \operatorname{tr}\left(\left(\overline{\boldsymbol{\Lambda}}_{i j k}-z \mathbf{I}_{M}\right)^{-1}\right)\right)^{2}} \\
& \mathrm{E}_{\mathbf{t}_{D i j}, \overline{\boldsymbol{\Lambda}}_{i j}}\left[\sum_{l=1}^{M} \frac{\left|t_{D i j l}\right|^{2}}{\left(\bar{\lambda}_{i j l}-z\right)^{2}}\right]
\end{aligned}
$$

where $\mathbf{t}_{D i j}=\left[t_{D i j 1}, t_{D i j 2}, t_{D i j 3}, \cdots, t_{D i j M}\right]$ and $\overline{\boldsymbol{\Lambda}}_{i j}=$ $\left[\bar{\lambda}_{i j 1}, \bar{\lambda}_{i j 2}, \cdots, \bar{\lambda}_{i j M}\right]$. Now, $\frac{1}{M} \operatorname{tr}\left(\left(\overline{\boldsymbol{\Lambda}}_{i j k}-z \mathbf{I}_{M}\right)^{-1}\right)$ can be represented by the Stieltjes transform $m_{F^{\prime}} \bar{\Lambda}_{i j k}(z)$ for large $M$. The CDF of the entries of $M^{\frac{\alpha}{2}} \overline{\mathbf{D}}_{i j k}$ can be proved to converge almost surely to (45) in the asymptotic regime considering that the infinite network is centered around the $i$-th BS. Furthermore, $\overline{\mathbf{H}}_{i j k} \in \mathbb{C}^{M \times(K-2)}$ contains iid complex entries with $\mathrm{E}\left[\overline{\mathbf{H}}_{i j k 11}-\mathrm{E}\left[\overline{\mathbf{H}}_{i j k 11}\right]\right]^{2}=1$. Therefore, according to the theorem of Silverstein in [25], ESD of $\frac{1}{M} \overline{\mathbf{H}}_{i j k} M^{\frac{\alpha}{2}} \overline{\mathbf{D}}_{i j k} \overline{\mathbf{H}}_{i j k}^{H}$, denoted as $F^{\overline{\mathbf{\Lambda}}_{i j k}}$ converges in distribution to a non-random function $F_{l} \overline{\boldsymbol{\Lambda}}^{i j k}$. This implies that the Stieltjes transform $m_{F^{\mathbf{\Lambda}_{i j k}}}$ also converges to a non-random function $m_{F_{l} \overline{\boldsymbol{\Lambda}}_{i j k}}$, which can be shown following the proof of Theorem 1 to be the unique, non-negative real solution of (38) where $z=-\frac{M^{\frac{\alpha}{2}-1} \sigma^{2} u_{i}}{P_{t}}$ and $\bar{m}_{i j k}$ is used instead of $m_{F_{l}} \overline{\bar{i}}_{i j k}$ for notational simplicity. Therefore, (68) can be written as

$$
\left|\mathbf{h}_{i k}^{H} \mathbf{w}_{i j}\right|^{2} \stackrel{a . r}{\rightarrow} \frac{\frac{\left|M^{\frac{-\alpha}{2}} \mathbf{t}_{i k}^{H}\left(\overline{\mathbf{\Lambda}}_{i j k}-z \mathbf{I}_{M}\right)^{-1} \mathbf{t}_{N i j}\right|^{2}}{\left(M^{-\frac{\alpha}{2}}+r_{i k}^{-\alpha} \bar{m}_{i j k}(z)\right)^{2}}}{\mathrm{E}_{\mathbf{t}_{D i j}, \overline{\boldsymbol{\Lambda}}_{i j}}\left[\sum_{l=1}^{M} \frac{\left|t_{D i j l}\right|^{2}}{\left(\bar{\lambda}_{i j l}-z\right)^{2}}\right]} .
$$




\section{Moreover}

$$
\begin{aligned}
& \left|M^{\frac{-\alpha}{2}} \mathbf{t}_{i k}^{H}\left(\overline{\mathbf{\Lambda}}_{i j k}-z \mathbf{I}_{M}\right)^{-1} \mathbf{t}_{N i j}\right|^{2} \\
& =M^{-\alpha} \mathbf{t}_{i k}^{H}\left(\overline{\mathbf{\Lambda}}_{i j k}-z \mathbf{I}_{M}\right)^{-1} \mathbf{t}_{N i j} \mathbf{t}_{N i j}^{H}\left(\overline{\boldsymbol{\Lambda}}_{i j k}-z \mathbf{I}_{M}\right)^{-1} \mathbf{t}_{i k} \\
& =M^{-\alpha} \sum_{m=1}^{M} \sum_{\substack{n=1 \\
n \neq m}}^{M} \frac{t_{i k m}^{H} t_{N i j m} t_{N i j n}^{H} t_{i k n}}{\left(\bar{\lambda}_{i j k m}-z\right)\left(\bar{\lambda}_{i j k n}-z\right)} \\
& \quad+M^{-\alpha} \sum_{l=1}^{M} \frac{\left|t_{i k l}\right|^{2}\left|t_{N i j l}\right|^{2}}{\left(\bar{\lambda}_{i j k l}-z\right)^{2}},
\end{aligned}
$$

where $\mathbf{t}_{i k}=\left[t_{i k 1}, \cdots, t_{i k M}\right], \mathbf{t}_{N i j}=\left[t_{N i j 1}, \cdots, t_{N i j M}\right]$ and $\overline{\boldsymbol{\Lambda}}_{i j k}=\left[\bar{\lambda}_{i j k 1}, \cdots, \bar{\lambda}_{i j k M}\right]$. Both $\mathbf{t}_{i k}$ and $\mathbf{t}_{N i j}$ are complex Gaussian vectors with mean 0 and covariance matrix $\mathbf{I}_{M}$. Moreover, they are independent of $\overline{\mathbf{U}}_{i j k}^{H}$ and $\overline{\boldsymbol{\Lambda}}_{i j k}$. Since $\mathbf{h}_{i k}$ and $\mathbf{h}_{i j}$ are independent, and $\mathbf{t}_{i k}=\overline{\mathbf{U}}_{i j k}^{H} \mathbf{h}_{i k}, \mathbf{t}_{N i j}=\overline{\mathbf{U}}_{i j k}^{H} \mathbf{h}_{i j}$, $\mathbf{t}_{N i j}$ and $\mathbf{t}_{i k}$ are also independent. Furthermore, the entries of $\mathbf{t}_{N i j}$ and $\mathbf{t}_{i k}$ are iid with mean 0 . Therefore, we can write

$$
\begin{aligned}
\sum_{m=1}^{M} \sum_{\substack{n=1 \\
n \neq m}}^{M} \mathrm{E}_{t, \lambda}\left[\frac{M^{-\alpha} t_{i k m}^{H} t_{N i j m} t_{N i j n}^{H} t_{i k n}}{\left(\bar{\lambda}_{i j k m}-z\right)\left(\bar{\lambda}_{i j k n}-z\right)}\right] \\
=\sum_{\substack { m=1 \\
\begin{subarray}{c}{n=1 \\
n \neq m{ m = 1 \\
\begin{subarray} { c } { n = 1 \\
n \neq m } }\end{subarray}}^{M} \mathrm{E}_{t, \lambda}\left[\frac{M^{-\alpha} t_{i k m}^{H} t_{N i j m} t_{N i j n}^{H}}{\left(\bar{\lambda}_{i j k m}-z\right)\left(\bar{\lambda}_{i j k n}-z\right)}\right] \mathrm{E}\left[t_{i k n}\right] \\
=0
\end{aligned}
$$

Using (71) and (70), we can write

$$
\begin{aligned}
\mathrm{E}_{\mathbf{t}_{i k}, \mathbf{t}_{N i j}, \overline{\mathbf{\Lambda}}_{i j k}} & {\left[\left|M^{-\frac{\alpha}{2}} \mathbf{t}_{i k}^{H}\left(\overline{\boldsymbol{\Lambda}}_{i j k}-z \mathbf{I}_{M}\right)^{-1} \mathbf{t}_{N i j}\right|^{2}\right] } \\
& =\mathrm{E}_{\mathbf{t}_{i k}, \mathbf{t}_{N i j}, \overline{\boldsymbol{\Lambda}}_{i j k}}\left[\sum_{l=1}^{M} \frac{M^{-\alpha}\left|t_{i k l}\right|^{2}\left|t_{N i j l}\right|^{2}}{\left(\bar{\lambda}_{i j k l}-z\right)^{2}}\right] .
\end{aligned}
$$

Using (72) and (69), mean of $\left|\mathbf{h}_{i k}^{H} \mathbf{w}_{i j}\right|^{2}$ conditioned on $r_{0 k}$ and $r_{i k}$ can be written as

$$
\begin{aligned}
\mathrm{E}_{\mathbf{h}_{i k}, \mathbf{w}_{i j}}\left[\left.\left|\mathbf{h}_{i k}^{H} \mathbf{w}_{i j}\right|^{2}\right|_{r_{0 k}, r_{i k}}\right] & \mathrm{E}_{\mathbf{t}_{i k}, \mathbf{t}_{N i j}, \overline{\mathbf{\Lambda}}_{i j k}\left[\sum_{l=1}^{M} \frac{M^{-\alpha}\left|t_{i k l}\right|^{2}\left|t_{N i j l}\right|^{2}}{\left(\bar{\lambda}_{i j k l}-z\right)^{2}}\right]} \stackrel{\text { a.r. }}{\left(M^{-\frac{\alpha}{2}}+r_{i k}^{-\alpha} \bar{m}_{i j k}(z)\right)^{2} \mathrm{E}_{\mathbf{t}_{D i j}, \overline{\mathbf{\Lambda}}_{i j}}\left[\sum_{l=1}^{M} \frac{\left|t_{D i j l}\right|^{2}}{\left(\bar{\lambda}_{i j l}-z\right)^{2}}\right]} .
\end{aligned}
$$

Note that $\left|t_{i k l}\right|^{2},\left|t_{N i j l}\right|^{2},\left(\bar{\lambda}_{i j k l}-z\right)^{-2}$ are independent to each other with $\mathrm{E}\left[\left|t_{i k l}\right|^{2}\right]=\mathrm{E}\left[\left|t_{N i j l}\right|^{2}\right]=1$. Moreover, $\left|t_{D i j l}\right|^{2}$ and $\left(\bar{\lambda}_{i j l}-z\right)^{-2}$ are independent to each other with $\mathrm{E}\left[\left|t_{D i j l}\right|^{2}\right]=1$. Therefore, (73) can be written as

$$
\begin{aligned}
& \mathrm{E}_{\mathbf{h}_{i k}, \mathbf{w}_{i j}}\left[\left.\left|\mathbf{h}_{i k}^{H} \mathbf{w}_{i j}\right|^{2}\right|_{r_{0 k}, r_{i k}}\right] \\
& \stackrel{a . r_{\dot{m}}}{\longrightarrow} \frac{\mathrm{E}_{\overline{\boldsymbol{\Lambda}}_{i j k}}\left[M^{-\alpha} \sum_{l=1}^{M} \frac{1}{\left(\bar{\lambda}_{i j k l}-z\right)^{2}}\right]}{\left(M^{-\frac{\alpha}{2}}+r_{i k}^{-\alpha} \bar{m}_{i j k}(z)\right)^{2} \mathrm{E}_{\overline{\boldsymbol{\Lambda}}_{i j}}\left[\sum_{l=1}^{M} \frac{1}{\left(\bar{\lambda}_{i j l}-z\right)^{2}}\right]} .
\end{aligned}
$$

$\bar{\lambda}_{i j l}$ are the eigenvalues of $\frac{1}{M} \overline{\mathbf{H}}_{i j} M^{\frac{\alpha}{2}} \overline{\mathbf{D}}_{i j} \overline{\mathbf{H}}_{i j}^{H}$, while $\bar{\lambda}_{i j k l}$ are the eigenvalues of $\frac{1}{M} \overline{\mathbf{H}}_{i j k} M^{\frac{\alpha}{2}} \overline{\mathbf{D}}_{i j k} \overline{\mathbf{H}}_{i j k}^{H}$, which is the rank1 perturbation $\left(-M^{\frac{\alpha}{2}-1} r_{i k}^{-\alpha} \mathbf{h}_{i k} \mathbf{h}_{i k}^{H}\right)$ of $\frac{1}{M} \overline{\mathbf{H}}_{i j} M^{\frac{\alpha}{2}} \overline{\mathbf{D}}_{i j} \overline{\mathbf{H}}_{i j}^{H}$. According to lemma 5 in [40], rank-1 perturbation does not affect the trace in the denominator in (74) for large $\mathrm{M}$. Therefore we have

$$
\lim _{M \rightarrow \infty} \sum_{l=1}^{M}\left(\bar{\lambda}_{i j l}-z\right)^{-2}=\lim _{M \rightarrow \infty} \sum_{l=1}^{M}\left(\bar{\lambda}_{i j k l}-z\right)^{-2} .
$$

Using (75), (74) can be expressed as

$$
\mathrm{E}_{\mathbf{h}_{i k}, \mathbf{w}_{i j}}\left[\left.\left|\mathbf{h}_{i k}^{H} \mathbf{w}_{i j}\right|^{2}\right|_{r_{0 k}, r_{i k}}\right] \stackrel{a . r_{\dot{p}}}{\longrightarrow} \frac{1}{\left(1+M^{\frac{\alpha}{2}} r_{i k}^{-\alpha} \bar{m}_{i j k}(z)\right)^{2}} .
$$

This completes the proof.

\section{Proof of Theorem 4}

The mean inter-cell interference power conditioned on $r_{0 k}$ can be written as

$$
\begin{aligned}
\mathrm{E}_{\mathbf{h}_{i k}, \mathbf{w}_{i j}, r_{i k}} & {\left[\left.\sum_{u=1}^{u_{\max }} \sum_{i \in \Phi_{u} \backslash\{0\}} \frac{P_{t} r_{i k}^{-\alpha}}{u} \sum_{j=1}^{u}\left|\mathbf{h}_{i k}^{H} \mathbf{w}_{i j}\right|^{2}\right|_{r_{0 k}}\right] } \\
& =\sum_{u=1}^{u_{\max }} \mathrm{E}_{r_{i k}}\left[\sum_{i \in \Phi_{u} \backslash\{0\}} \frac{P_{t} r_{i k}^{-\alpha}}{u} \sum_{j=1}^{u} \mathrm{E}_{\mathbf{h}_{i k}, \mathbf{w}_{i j}}\left[\left.\left|\mathbf{h}_{i k}^{H} \mathbf{w}_{i j}\right|^{2}\right|_{r_{i k}, r_{0 k}}\right]\right] .
\end{aligned}
$$

$\mathrm{E}_{\mathbf{h}_{i k}, \mathbf{w}_{i j}}\left[\left.\left|\mathbf{h}_{i k}^{H} \mathbf{w}_{i j}\right|^{2}\right|_{r_{i k}, r_{0 k}}\right]=\mathrm{E}_{\mathbf{h}_{i k}, \mathbf{w}_{i j^{\prime}}}\left[\left.\left|\mathbf{h}_{i k}^{H} \mathbf{w}_{i j^{\prime}}\right|^{2}\right|_{r_{i k}, r_{0 k}}\right]$ $\forall i, \forall j \neq j^{\prime}$, and hence (77) can be written using Theorem 3 as

$$
\begin{aligned}
\mathrm{E}_{\mathbf{h}_{i k}, \mathbf{w}_{i j}, r_{i k}} & {\left[\left.\sum_{u=1}^{u_{\max }} \sum_{i \in \Phi_{u} \backslash\{0\}} \frac{P_{t} r_{i k}^{-\alpha}}{u} \sum_{j=1}^{u}\left|\mathbf{h}_{i k}^{H} \mathbf{w}_{i j}\right|^{2}\right|_{r_{0 k}}\right] } \\
& \stackrel{a . r_{.}}{\longrightarrow} \sum_{u=1}^{u_{\max }} \mathrm{E}_{r_{i k}}\left[\sum_{i \in \Phi_{u} \backslash\{0\}} \frac{P_{t} r_{i k}^{-\alpha}}{\left(1+M^{\frac{\alpha}{2}} r_{i k}^{-\alpha} \bar{m}_{i j k}(z)\right)^{2}}\right] .
\end{aligned}
$$

Using the Campbell's theorem for the PPP $\Phi_{u}$ [22], we can write

$$
\begin{aligned}
\mathrm{E}_{r_{i k}} & {\left[\sum_{i \in \Phi_{u} \backslash\{0\}} \frac{P_{t} r_{i k}^{-\alpha}}{\left(1+M^{\frac{\alpha}{2}} r_{i k}^{-\alpha} \bar{m}_{i j k}(z)\right)^{2}}\right] } \\
& =2 \pi \lambda_{b} p_{N}(u) \int_{r_{0 k}}^{\infty} \frac{P_{t} r_{i k}^{1-\alpha}}{\left(1+M^{\frac{\alpha}{2}} r_{i k}^{-\alpha} \bar{m}_{i j k}(z)\right)^{2}} d r_{i k} .
\end{aligned}
$$


Using $\tau=M^{\frac{\alpha}{2}} r_{i k}^{-\alpha}$, (79) can be written as

$$
\begin{aligned}
\mathrm{E}_{r_{i k}} & {\left[\sum_{i \in \Phi_{u} \backslash\{0\}} \frac{P_{t} r_{i k}^{-\alpha}}{\left(1+M^{\frac{\alpha}{2}} r_{i k}^{-\alpha} \bar{m}_{i j k}(z)\right)^{2}}\right] } \\
& =2 \pi \lambda_{b} p_{N}(u) \int_{M^{\frac{\alpha}{2}} r_{0 k}^{-\alpha}}^{0} \frac{P_{t}\left(\tau^{-\frac{1}{\alpha}} M^{\frac{1}{2}}\right)^{(1-\alpha)} \frac{-1}{\alpha} \tau^{-\frac{1}{\alpha}-1} M^{\frac{1}{2}}}{\left(1+\tau \bar{m}_{i j k}(z)\right)^{2}} d \tau \\
& =-\frac{2 \pi \lambda_{b} p_{N}(u) P_{t} M^{1-\frac{\alpha}{2}}}{\alpha} \int_{M^{\frac{\alpha}{2}} r_{0 k}^{-\alpha}}^{0} \frac{\tau^{-\frac{2}{\alpha}}}{\left(1+\tau \bar{m}_{i j k}(z)\right)^{2}} d \tau \\
& =f_{1}(u)-f_{2}\left(u, r_{0 k}\right),
\end{aligned}
$$

with $f_{1}(u)$ and $f_{2}\left(u, r_{0 k}\right)$ as in Theorem 4. Using (80), (78) can be written as,

$$
\begin{aligned}
\mathrm{E}_{\mathbf{h}_{i k}, \mathbf{w}_{i j}, r_{i k}} & {\left[\left.\sum_{u=1}^{u_{\max }} \sum_{i \in \Phi_{u} \backslash\{0\}} \frac{P_{t} r_{i k}^{-\alpha}}{u} \sum_{j=1}^{u}\left|\mathbf{h}_{i k}^{H} \mathbf{w}_{i j}\right|^{2}\right|_{r_{0 k}}\right] } \\
& \stackrel{a . r .}{\longrightarrow} \sum_{u=1}^{u_{\max }}\left(f_{1}(u)-f_{2}\left(u, r_{0 k}\right)\right)
\end{aligned}
$$

and the proof is complete.

\section{REFERENCES}

[1] X. Hong, Y. Jie, C.-X. Wang, J. Shi, and X. Ge, "Energy-spectral efficiency trade-off in virtual MIMO cellular systems," IEEE Journal on Selected Areas in Communications, vol. 31, no. 10, pp. 2128-2140, 2013.

[2] J. M. Gorce, D. Tsilimantos, P. Ferrand, and V. H. Poor, "EnergyCapacity Trade-off Bounds in a Downlink Typical Cell," in IEEE 25th International Symposium on Personal Indoor and Mobile Radio Communications (PIMRC), September 2014, pp. 1-6.

[3] D. Cao, S. Zhou, and Z. Niu, "Optimal Combination of Base Station Densities for Energy-Efficient Two-Tier Heterogeneous Cellular Networks," IEEE Transactions on Wireless Communications, vol. 12, no. 9 , pp. 4350-4362, September 2013.

[4] M. Maaz, J. Lorandel, P. Mary, J.-C. Prévotet, and M. Hélard, "Energy efficiency analysis of hybrid-ARQ relay-assisted schemes in LTE-based systems," EURASIP Journal on Wireless Communications and Networking, no. 1, pp. 1-13, 2016.

[5] M. Imran, J. Alonso-Rubio, G. Auer, M. Boldi, M. Braglia, P. Fazekas, D. Ferling, A. Fehske, P. Frenger, R. Gupta et al., "Most suitable efficiency metrics and utility functions," EARTH Project Report, pp. $1-89,2011$.

[6] C. Sun and C. Yang, "Energy efficiency analysis of one-way and twoway relay systems," EURASIP Journal on Wireless Communications and Networking, no. 1, pp. 1-18, 2012.

[7] V. G. Auer and O. Blume, "Energy efficiency analysis of the reference systems, areas of improvements and target breakdown (earth)," 2012.

[8] C. Desset, B. Debaillie, V. Giannini, A. Fehske, G. Auer, H. Holtkamp, W. Wajda, D. Sabella, F. Richter, M. J. Gonzalez et al., "Flexible power modeling of LTE base stations," in IEEE Wireless Communications and Networking Conference (WCNC), 2012, pp. 2858-2862.

[9] H. Q. Ngo, E. G. Larsson, and T. L. Marzetta, "Energy and spectral efficiency of very large multiuser MIMO systems," IEEE Transactions on Communications, vol. 61, no. 4, pp. 1436-1449, 2013.

[10] C. Li, J. Zhang, and K. Letaief, "Performance analysis of SDMA in multicell wireless networks," in IEEE Global Communications Conference (GLOBECOM), 2013, pp. 3867-3872.

[11] A. K. Gupta, H. S. Dhillon, S. Vishwanath, and J. G. Andrews, "Downlink coverage probability in MIMO HetNets with flexible cell selection," in IEEE Global Communications Conference (GLOBECOM), 2014, pp. 1534-1539.

[12] H. S. Dhillon, M. Kountouris, and J. G. Andrews, "Downlink MIMO HetNets: Modeling, ordering results and performance analysis," IEEE Transactions on Wireless Communications, vol. 12, no. 10, pp. 5208$5222,2013$.
[13] P. Cheng, M. Tao, and W. Zhang, "A new SLNR-based linear precoding for downlink multi-user multi-stream MIMO systems," IEEE Cотmunications Letters, vol. 14, no. 11, pp. 1008-1010, 2010.

[14] E. Bjornson, M. Bengtsson, and B. Ottersten, "Optimal multiuser transmit beamforming: A difficult problem with a simple solution structure [lecture notes]," IEEE Signal Processing Magazine, vol. 31, no. 4, pp. 142-148, 2014.

[15] M. Sadek, A. Tarighat, and A. H. Sayed, "A leakage-based precoding scheme for downlink multi-user MIMO channels," IEEE Transactions on Wireless Communications, vol. 6, no. 5, pp. 1711-1721, 2007.

[16] A. Tarighat, M. Sadek, and A. H. Sayed, "A multi user beamforming scheme for downlink MIMO channels based on maximizing signal-toleakage ratios," in IEEE International Conference on Acoustics, Speech, and Signal Processing (ICASSP'05), vol. 3, 2005.

[17] P. Patcharamaneepakorn, S. Armour, and A. Doufexi, "On the Equivalence Between SLNR and MMSE Precoding Schemes with SingleAntenna Receivers," IEEE Communications Letters, vol. 16, no. 7, pp. 1034-1037, July 2012.

[18] B. O. Lee, H. W. Je, I. Sohn, O. S. Shin, and K. B. Lee, "Interferenceaware decentralized precoding for multicell mimo tdd systems," in IEEE GLOBECOM 2008 - 2008 IEEE Global Telecommunications Conference, Nov 2008, pp. 1-5.

[19] R. Stridh, M. Bengtsson, and B. Ottersten, "System evaluation of optimal downlink beamforming with congestion control in wireless communication," IEEE Transactions on Wireless Communications, vol. 5, no. 4, pp. 743-751, 2006.

[20] D. Gesbert and R. Zakhour, "Coordination on the miso interference channel using the virtual sinr framework," in Workshop on Smart Antennas (WSA), vol. 17, 2009, pp. 1-18.

[21] J. G. Andrews, F. Baccelli, and R. K. Ganti, "A tractable approach to coverage and rate in cellular networks," IEEE Transactions on Communications, vol. 59, no. 11, pp. 3122-3134, 2011.

[22] M. Haenggi and R. K. Ganti, Interference in large wireless networks. Now Publishers Inc, 2009.

[23] A. M. Alam, P. Mary, J.-Y. Baudais, and X. Lagrange, "Energy Efficiency-Area Spectral Efficiency Tradeoff in PPP Network with SLNR Precoder," in the 17th IEEE International Workshop on Signal Processing Advances in Wireless Communications (SPAWC), vol. 17, 2016, pp. 1-6.

[24] E. Bjornson, L. Sanguinetti, and M. Kountouris, "Designing wireless broadband access for energy efficiency: Are small cells the only answer?" in IEEE International Conference on Communication Workshop (ICCW), 2015, pp. 136-141.

[25] J. W. Silverstein, "Strong convergence of the empirical distribution of eigenvalues of large dimensional random matrices," Journal of Multivariate Analysis, vol. 55, no. 2, pp. 331-339, 1995.

[26] D. N. C. Tse and S. V. Hanly, "Linear multiuser receivers: effective interference, effective bandwidth and user capacity," IEEE Transactions on Information Theory, vol. 45, no. 2, pp. 641-657, March 1999.

[27] P. Li, D. Paul, R. Narasimhan, and J. Cioffi, "On the distribution of SINR for the MMSE MIMO receiver and performance analysis," IEEE Transactions on Information Theory, vol. 52, no. 1, pp. 271-286, 2006.

[28] J. Ma, Y. J. Zhang, X. Su, and Y. Yao, "On capacity of wireless ad hoc networks with MIMO MMSE receivers," IEEE Transactions on Wireless Communications, vol. 7, no. 12, pp. 5493-5503, December 2008.

[29] S. Govindasamy, D. W. Bliss, and D. H. Staelin, "Spectral Efficiency in Single-Hop Ad-Hoc Wireless Networks with Interference Using Adaptive Antenna Arrays," IEEE Journal on Selected Areas in Communications, vol. 25, no. 7, pp. 1358-1369, September 2007.

[30] G. Auer, O. Blume, V. Giannini, I. Godor, M. Imran, Y. Jading, E. Katranaras, M. Olsson, D. Sabella, P. Skillermark et al., "D2. 3: Energy efficiency analysis of the reference systems, areas of improvements and target breakdown," EARTH, 2010.

[31] C. Li, J. Zhang, and K. Letaief, "Throughput and energy efficiency analysis of small cell networks with multi-antenna base stations," IEEE Transactions on Wireless Communications, vol. 13, no. 5, pp. 25052517, 2014.

[32] P. Calka, "Precise formulae for the distributions of the principal geometric characteristics of the typical cells of a two-dimensional poissonvoronoi tessellation and a poisson line process," Advances in Applied Probability, vol. 35, no. 03, pp. 551-562, 2003.

[33] J.-S. Ferenc and Z. Néda, "On the size distribution of Poisson Voronoi cells," Physica A: Statistical Mechanics and its Applications, vol. 385, no. 2, pp. 518-526, 2007.

[34] G. H. Golub and C. F. Van Loan, Matrix computations. JHU Press, 2012, vol. 3 . 
[35] F. Boccardi and H. Huang, "Zero-forcing precoding for the MIMO broadcast channel under per-antenna power constraints," in IEEE 7th Workshop on Signal Processing Advances in Wireless Communications (SPAWC), 2006, pp. 1-5.

[36] G. George, R. K. Mungara, A. Lozano, and M. Haenggi, "Ergodic spectral efficiency in mimo cellular networks," arXiv preprint arXiv:1607.04352, 2016.

[37] M. Di Renzo, A. Guidotti, and G. E. Corazza, "Average rate of downlink heterogeneous cellular networks over generalized fading channels: A stochastic geometry approach," IEEE Transactions on Communications, vol. 61, no. 7, pp. 3050-3071, 2013.

[38] L. Yu, W. Liu, and R. Langley, "SINR Analysis of the Subtraction-Based SMI Beamformer," IEEE Transactions on Signal Processing, vol. 58, no. 11, pp. 5926-5932, Nov 2010

[39] M. Abramowitz and I. Stegun, Handbook of mathematical functions: with formulas, graphs, and mathematical tables. Courier Corporation, 1964, vol. 55.

[40] R. Couillet, M. Debbah, and J. W. Silverstein, "A Deterministic Equivalent for the Analysis of Correlated MIMO Multiple Access Channels," IEEE Transactions on Information Theory, vol. 57, no. 6, pp. 34933514, June 2011.

[41] A. M. Alam, P. Mary, J.-Y. Baudais, and X. Lagrange, "Energy Efficiency-Spectral Efficiency Tradeoff in Interference-Limited Wireless Networks with Shadowing," in IEEE 82nd Vehicular Technology Conference: VTC2015-Fall, vol. 82, 2015, pp. 1-5.

[42] D. B. Cheikh, J.-M. Kelif, M. Coupechoux, and P. Godlewski, "Multicellular zero forcing precoding performance in Rayleigh and shadow fading," in IEEE 73rd Vehicular Technology Conference (VTC Spring), 2011, pp. $1-5$.

[43] A. Guionnet, O. Zeitouni et al., "Concentration of the spectral measure for large matrices," Electron. Comm. Probab, vol. 5, pp. 119-136, 2000.

[44] J. W. Silverstein and Z. Bai, "On the empirical distribution of eigenvalues of a class of large dimensional random matrices," Journal of Multivariate analysis, vol. 54, no. 2, pp. 175-192, 1995.

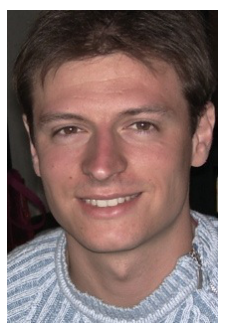

Philippe Mary (S'06-M'09) received his M.Sc. in Signal Processing and Digital Communications from the University of Nice Sophia-Antipolis and the Dipl. Ing. degree in electrical engineering from the Polytechnic University School of Nice SophiaAntipolis (France) both in 2004. He received his $\mathrm{PhD}$ in electrical engineering from the National Institute of Applied Sciences of Lyon in 2008. During his $\mathrm{PhD}$, he was with France Telecom $\mathrm{R} \& \mathrm{D}$ and he worked on the analytical performance study for mobile communications considering shadowing and fading and multi-user detectors for wireless communications. From 2008 to 2009, he was post-doctoral researcher at ETIS Laboratory in Cergy-Pontoise.

In September 2009, Dr. Mary joined the Digital Communication Systems department of INSA Rennes and IETR laboratory as associate professor. In 2011, he served as TPC chair for the third international workshop on cross-layer design (IWCLD 2011) and he is serving as TPC member of various IEEE conferences, e.g. ICC, Globecom, PIMRC, WCNC, VTC. His research interests include analytical performance analysis and signal processing for digital communications, resource allocation and finite block length communication theory.

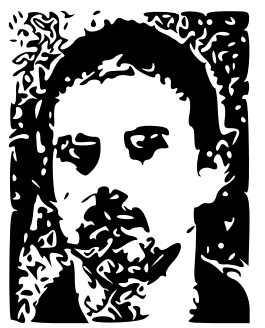

Jean-Yves Baudais received the M.Sc. degree, and $\mathrm{PhD}$ degree in electrical engineering from the National Institute of Applied Sciences of Rennes (INSA), France, in 1997 and 2001 respectively. In 2002, he joined the French National Centre for Scientific Research (CNRS), where he is now researcher in the Institute for Electronics and Telecommunications of Rennes (IETR), Communication systems (SYSCOM) team. In 2014, he received the "habilitation à diriger les recherches" from the University of Rennes. His general interests lie in the areas of signal processing and digital communications: transmitter design, resource allocation, receiver diversity processing (space, time, frequency), multiuser and multicarrier processing, network performance measure with point process model, energetic performance evaluation and alos radar signal processing.

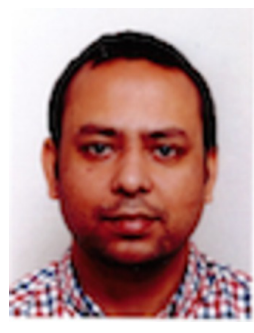

Ahmad Mahbubul Alam received his B.Sc degree with distinction in Electrical and Electronic Engineering from Islamic University of Technology (IUT), Bangladesh in 2007. Afterward, he worked for Huawei Technologies Bangladesh Ltd. as a base station subsystem (BSS) engineer and as a lecturer in Stamford University Bangladesh. He obtained his M.Sc degree with distinction in Communications Engineering from Aalto University, Finland in 2013. Between 2010 and 2013, he worked as a summer intern, research assistant and teaching assistant at Aalto University. His research activities during this period were mainly focused on the prediction of signal strength in order to estimate the available white space in the licensed TV band. He received his $\mathrm{PhD}$ degree in Electronic and Telecommunications from INSA de Rennes, France in 2017. His current research interests include energy efficiency-spectral efficiency (EE-SE) tradeoff in cellular network, multi-user multiple input single output (MU-MISO) system, stochastic architecture for cellular network, precoding techniques.

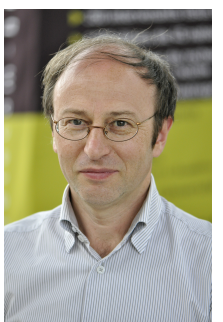

Xavier Lagrange received the Engineering Degree from Ecole Centrale des Arts et Manufactures, Paris, France, in 1984 and the Ph.D. degree from TELECOM Paristech in 1998. Since 2002, he has been professor in IMT Atlantique (formerly known as Telecom Bretagne) at the "Network Systems, Cybersecurity and Digital Law". He leads research group ADOPNET (Advanced Technologies for Operated Networks) at IRISA (Institut de Recherche en Informatique et Systémes Aléatoires). His domain of interest includes resource allocation, medium access control and performance analysis for $4 \mathrm{G}$ and $5 \mathrm{G}$ cellular networks. He is a senior member of IEEE. 